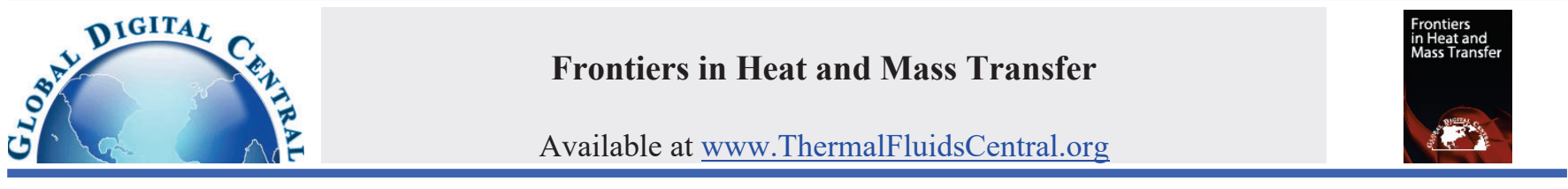

\title{
COMPREHENSIVE EXAMINATION OF THE THREE-DIMENSIONAL ROTATING FLOW OF A UCM NANOLIQUID OVER AN EXPONENTIALLY STRETCHABLE CONVECTIVE SURFACE UTILIZING THE OPTIMAL HOMOTOPY ANALYSIS METHOD
}

\author{
K.V. Prasad ${ }^{a}$, Hanumesh Vaidya ${ }^{a}{ }^{*}$, O. D. Makinde ${ }^{b}$, K. Vajravelu ${ }^{c}$, A. Wakif ${ }^{\mathrm{d}}$, Hussain Basha ${ }^{\mathrm{a}}$ \\ ${ }^{a}$ Department of Mathematics, VSK University, Vinayaka Nagar, Ballari-583 105, Karnataka, India \\ ${ }^{b}$ Faculty of Military Science, Stellenbosch University, Private Bag X2, Saldanha 7395, South Africa \\ ${ }^{c}$ Department of Mathematics, University of Central Florida, Orlando, FL 32816, USA \\ ${ }^{d}$ Hassan II University, Faculty of Sciences Aïn Chock, Laboratory of Mechanics, B.P.5366 Mâarif, Casablanca, Morocco.
}

\begin{abstract}
This article explores the three-dimensional (3D) rotating flow of Upper Convected Maxwell (UCM) nanoliquid over an exponentially stretching sheet with a convective boundary condition and zero mass flux for the nanoparticles concentration. The impacts of velocity slip and hall current are being considered. The suitable similarity transformations are employed to reduce the governing partial differential equations into ordinary ones. These systems of equations are highly non-linear, coupled and in turn solved by an efficient semi-analytical scheme known as optimal homotopy analysis method (OHAM). The effects of various physical constraints on velocity, temperature, and concentration fields are analyzed graphically and discussed in detail. The impact of hall current is reduced the temperature field whereas increase to the velocity and the concentration fields. The present results are compared with the available results in the literature to check the legitimacy of the present semianalytical scheme and noted an excellent agreement for limiting cases.
\end{abstract}

Keywords: Rotating flow, Thermal diffusivity, Nanoparticles diffusivity, Hall effects, OHAM.

\section{INTRODUCTION}

Boundary layer flow of a viscous fluid due to an impulsive motion over an elastic surface is involved in several areas of science and technology such as "drawing, annealing, and tinning of copper wires, rolling and manufacturing of plastic films, and artificial fibers," etc. In these application processes, the end product primarily depends on the rate of stretching of the surface, which is very significant. The pioneering work of Sakiadis (1961) considering stationary ambient fluid over a moving plate has brought new dimensions to the boundary layer theory. Crane (1970) obtained the closed-form of the exact solution for the velocity distribution and examined the work of Sakiadis (1961) by considering the velocity of the stretching sheet which is proportional to the distance from the slit. Rajagopal et al. (1984) made a comparative analysis between viscoelastic fluid and viscous fluid and examined the rate of cooling of viscoelastic fluid flow over a stretching sheet. Grubka and Bobba (1985) and Lawrence and Rao (1992) extended the work of Rajagopal et al. (1984) for heat transfer characteristics. Further, several researchers have examined the stretching sheet geometry problems for two-dimensional flows (Ganji et al. (2014); Parand et al. (2017); Rahimi et al. (2017) and three-dimensional flows (Hayat et al. (2012); Nadeem et al. (2013); Weidman and Ishak (2015)).

All the above studies confined their examinations to twodimensional/three-dimensional flows over linearly stretched sheets. On the other hand, the demands of the technological industries and previously mentioned applications are not only confined to a linearly stretched sheet but also to the non-linearly extruded sheet. The stretching of the sheet exponentially is one of the prominent methods to meet the nonlinear requirements of the industry. Given this, Magyari and Keller (1999) and Elbashbeshy (2001) analyzed the flow pattern due to an exponentially continuous stretching sheet.Sajid and Hayat (2008) and Bidin and Nazar (2009) employed HAM/Keller-box method to obtain the analytical/numerical solution to examine the impact of thermal radiation on the flow using exponentially stretching sheet. Further, Swati Mukhopadhyay (2013) investigated that the slip effects over a magnetic flow field with suction/blowing are prominent. Of late, Fazle et al. (2017) and Srinivasacharya and Jagadeeshwar (2017) extended the work of Magyari and Keller (1999) and Swati Mukhopadhyay (2013) by considering radiation effects.

In recent advancement, the nanotechnology is an attractive area of discussion owing to its enriching characteristics of controlling the thermal conductivity. A blend (Solid-Liquid) of very small-sized nanoparticle $(<100 \mathrm{~nm})$ and base fluid is known as nanofluid. The colloids of the base fluids are usually made up of metal and oxides, which enhance both the conduction and convection coefficient and also improve significantly the heat transfer rates of the coolants. The nanofluids have emerged as special kinds of many applications in heat transfer such as nuclear reactor cooling, solar water heating, domestic refrigerators, drag reduction, and thermal energy storage, etc. Choi (1995) has initially experimented on the base fluid, which is the addition of the mixture of metal oxides to the base fluid and observed the enhancement of the thermal properties in the fluid. Buongiorno (2006) proposed a most conventional model to describe the convective transport based on the mechanism of Brownian motion with thermophoretic diffusion and remarked that the heat transfer performance has a vital role in the nanofluid. Further, Tiwari and Das (2007) examined two-sided lid-driven differentially heated square cavity filled with nanofluids model which different form conventional Buongiorno (2006) model and explained behavior of particle size, momentum/thermal diffusivity, and temperature. Some recent attempts to describe in this direction are Mustafa et al. (2016); Hayat et al. (2017); Animasaun et al. (2019); Prasad et al. (2018);

\footnotetext{
* Corresponding author.

E-mail:hanumeshvaidya@gmail.com (Hanumesh Vaidya).
} 
Vaidya et al. (2019a, 2019b); Majdi et al. (2019); Puroshotaman et al.(2019) and Amini et al. (2020).

Inspired by subsequent developments in the available literature, our main objective of the present investigation is to analyze the threedimensional rotating flow of a UCM nanoliquid over an exponentially stretchable surface. Rotating flows usually involve in an anticyclone flow circulation, geological stretching of tectonic plate beneath the rotating ocean, centrifugal filtration process, in rotorstator systems, and cooling of skins of high-speed aircraft. The analysis is carried out in the presence of hall effect, velocity slip, convective boundary condition, and zero mass flux nanoparticle concentration. Here, the local similarity equations are derived and solved analytically for varying values of embedded parameters by the semi-analytical technique known as OHAM (see for details, Liao (2010); Marinca and Herisanu (2015); and Van Gorder (2019)). The impacts of different physical parameters on velocity, temperature, and concentration profiles are analyzed graphically. In addition to this, estimations of skin friction, local Nusselt number, and local Sherwood number are presented in the analysis, which is very important from the industrial application point of view.

\section{MATHEMATICAL FORMULATION AND PHYSICAL DESCRIPTION OF THE STUDIED FLOW PROBLEM}

Let's consider a steady three-dimensional (3D) rotating flow of a viscous incompressible Upper Convected Maxwell (UCM) nanoliquid by an exponentially stretchable surface subjected to the slip velocity, convective boundary condition and zero mass flux concentration. The Cartesian coordinate system is adopted in such a way that the surface is aligned with $x$ and $y$-axes and the fluid is taken in the space $z \geq 0$ (see Fig. 1).

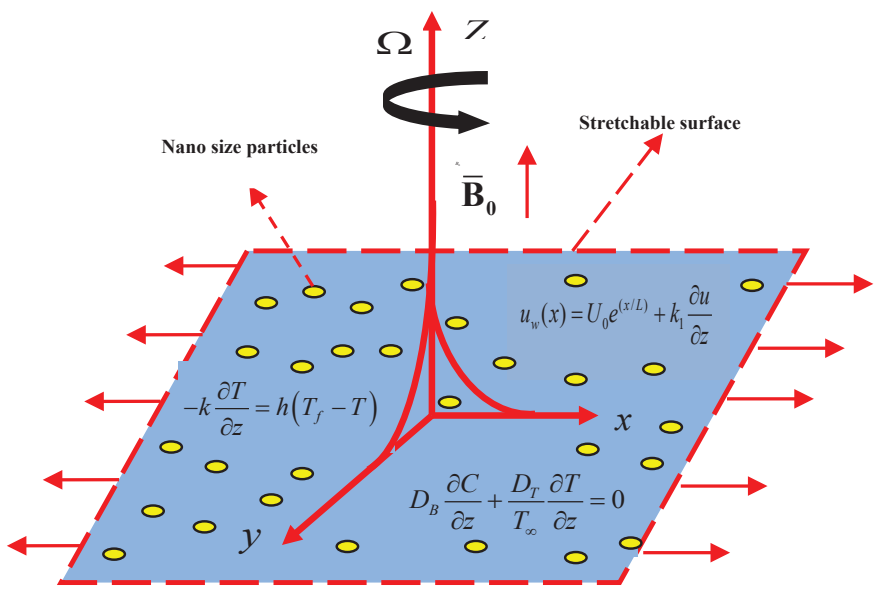

Fig. 1 Geometry of the Maxwell nanoliquid flow model.

The fluid is rotating about $Z$-axis with constant angular velocity $\Omega$. The fluid is considered electrically conducting, and a transverse magnetic field $\mathbf{B}_{0}$ is applied in the $Z$-direction. Further, the hall current effect is taken into account. In general, the hall current and electrically conducting fluid affect the flow in the presence of a strong magnetic field. The effect of hall current gives rise to a force in the $z$-direction, and hence the flow becomes three dimensional. The generalized Ohm's law with hall current is defined as

$\mathbf{J}=\sigma\left[\mathbf{E}+\mathbf{V} \times \mathbf{B}-\frac{1}{e n_{e}} \mathbf{J} \times \mathbf{B}+\frac{1}{e n_{e}} \Delta p_{e}\right]$

where $\boldsymbol{J}=\left(\boldsymbol{J}_{\boldsymbol{x}}, \boldsymbol{J}_{\boldsymbol{y}}, \boldsymbol{J}_{z}\right)$ is the current density vector, $\sigma$ is the electrical conductivity, $\mathbf{E}$ is the induced electric field resulting from the charge separation, $\mathbf{V}=(u, \mathrm{v}, w)$ is the velocity vector, $\boldsymbol{B}=\left(0,0, \boldsymbol{B}_{0}\right)$ is the magnetic induction vector, $e$ is the electric charge, $n_{e}$ is the electron number density and $p_{e}$ is the electronic pressure. Furthermore, there is no applied or polarization voltage is imposed on the flow $\mathbf{E}=(0,0,0)$. For weakly ionized gases, ion slip effect and the electron pressure gradient are ignored. The generalized ohm's law under the above-mentioned conditions for electrically non-conducting sheet $\boldsymbol{J}_{z}=0$. Hence the Eq. (1) becomes

$\left\{\mathbf{J}_{\mathbf{x}}=\frac{\sigma \mathbf{B}_{0}}{\left(1+m^{2}\right)}(m u-\mathrm{v}), \mathbf{J}_{\mathbf{y}}=\frac{\sigma \mathbf{B}_{0}}{\left(1+m^{2}\right)}(u+m \mathrm{v})\right.$.

Here, the following assumptions are considered.

a) Joule heating is neglected.

b) The wall is impermeable (i.e., $\left.\mathrm{v}_{\mathrm{w}}=0\right)$.

c) The sheet is stretchable with a variable velocity and slip velocity is given by $u_{w}(x)\left(=U_{0} e^{(x / L)}+k_{1} \frac{\partial u}{\partial z}\right)$, where $U_{o}$ is the reference velocity, $L$ is the characteristic length, $k_{1}$ is the slip constant. The physical problem under consideration includes the connections of momentum, energy, and mass. These relations can be condensed as pursue

$\frac{\partial u}{\partial x}+\frac{\partial \mathrm{v}}{\partial y}+\frac{\partial w}{\partial z}=0$
$u \frac{\partial u}{\partial x}+\mathrm{v} \frac{\partial u}{\partial y}+w \frac{\partial u}{\partial z}-2 \Omega \mathrm{v}$

$=v \frac{\partial^{2} u}{\partial z^{2}}-\lambda_{1}\left(\begin{array}{l}u^{2} \frac{\partial^{2} u}{\partial x^{2}}+v^{2} \frac{\partial^{2} u}{\partial y^{2}}+w^{2} \frac{\partial^{2} u}{\partial z^{2}}+2 u v \frac{\partial^{2} u}{\partial x \partial y}+2 \mathrm{v} w \frac{\partial^{2} u}{\partial y \partial z}+2 u w \frac{\partial^{2} u}{\partial x \partial z} \\ -2 \Omega\left(u \frac{\partial \mathrm{v}}{\partial x}+\mathrm{v} \frac{\partial \mathrm{v}}{\partial y}+w \frac{\partial \mathrm{v}}{\partial z}\right)+2 \Omega\left(\mathrm{v} \frac{\partial u}{\partial x}-u \frac{\partial u}{\partial y}\right)\end{array}\right)$ $-\frac{\sigma B_{0}^{2}}{\rho\left(1+m^{2}\right)}\left(u+m \mathrm{v} \lambda_{1} w \frac{\partial u}{\partial z}\right)$ $u \frac{\partial \mathrm{v}}{\partial x}+\mathrm{v} \frac{\partial \mathrm{v}}{\partial y}+w \frac{\partial \mathrm{v}}{\partial z}+2 \Omega u$

$=v \frac{\partial^{2} \mathrm{v}}{\partial z^{2}}-\lambda_{1}\left(\begin{array}{l}u^{2} \frac{\partial^{2} \mathrm{v}}{\partial x^{2}}+\mathrm{v}^{2} \frac{\partial^{2} \mathrm{v}}{\partial y^{2}}+w^{2} \frac{\partial^{2} \mathrm{v}}{\partial z^{2}}+2 u \mathrm{v} \frac{\partial^{2} \mathrm{v}}{\partial x \partial y}+2 \mathrm{v} w \frac{\partial^{2} \mathrm{v}}{\partial y \partial z}+2 u w \frac{\partial^{2} u}{\partial x \partial z} \\ +2 \Omega\left(u \frac{\partial u}{\partial x}+\mathrm{v} \frac{\partial u}{\partial y}+w \frac{\partial u}{\partial z}\right)+2 \Omega\left(\mathrm{v} \frac{\partial \mathrm{v}}{\partial x}-u \frac{\partial \mathrm{v}}{\partial y}\right)\end{array}\right)$ $-\frac{\sigma B_{0}^{2}}{\rho\left(1+m^{2}\right)}\left(m u \lambda_{1} w \frac{\partial \mathrm{v}}{\partial z}-\mathrm{v}\right)$,

$u \frac{\partial T}{\partial x}+\mathrm{v} \frac{\partial T}{\partial y}+w \frac{\partial T}{\partial z}=\alpha \frac{\partial^{2} T}{\partial z^{2}}+\tau\left(D_{B}\left(\frac{\partial T}{\partial z} \frac{\partial C}{\partial z}\right)+\frac{D_{T}}{T_{\infty}}\left(\frac{\partial T}{\partial z}\right)^{2}\right)$,

$u \frac{\partial C}{\partial x}+\mathrm{v} \frac{\partial C}{\partial y}+w \frac{\partial C}{\partial z}=D_{B}\left(\frac{\partial^{2} C}{\partial z^{2}}\right)+\frac{D_{T}}{T_{\infty}}\left(\frac{\partial^{2} T}{\partial z^{2}}\right)$,

with the following realistic boundary conditions (BCs)

$u=u_{w}(x)=U_{0} e^{(x / L)}+k_{1} \frac{\partial u}{\partial z}, \mathrm{v}=0$,

$w=0,-k \frac{\partial T}{\partial z}=h\left(T_{f}-T\right), D_{B} \frac{\partial C}{\partial z}+\frac{D_{T}}{T_{\infty}} \frac{\partial T}{\partial z}=0$ at $\mathrm{z}=0$,

$u \rightarrow 0, \quad \mathrm{v} \rightarrow 0, \quad T \rightarrow T_{\infty}, \quad C \rightarrow C_{\infty} \quad$ as $z \rightarrow \infty$.

Here $u, v$ and $w$ are the fluid velocity components along the $x, y$ and $z$-direction, respectively. Further, $v$ is the kinematic viscosity, $\lambda_{1}$ is the relaxation time, $\rho$ is the density of the fluid, $m$ is the hall effect parameter, $T$ is the temperature, $\alpha$ is the thermal diffusivity, $\tau$ is the ratio of the effective heat capacity of the nanoparticle material and heat capacity of the fluid, $D_{B}$ is the Brownian diffusion coefficient, $C$ is the concentration of nanoparticles, $D_{T}$ is the thermophoresis diffusion coefficient, $T_{\infty}$ is the ambient fluid temperature, $k$ is the thermal conductivity, $h$ is the heat transport coefficient, $T_{f}$ is the hot fluid temperature. The magnetic field $B_{0}$ is considered to be uniform. 


\subsection{Similarity variables and dimensionless governing equations}

To simplify the mathematical analysis of the model by the following similarity variables (see details Hayat et al. (2017)) are evoked,

$u=U_{0} e^{(x / L)} f^{\prime}(\zeta), v=U_{0} e^{(x / L)} g(\zeta), w=-\sqrt{\frac{v U_{0}}{2 L}} e^{(x / 2 L)}\left(f+\zeta f^{\prime}\right)$,

$T=T_{\infty}+\left(T_{f}-T_{\infty}\right) \theta(\zeta), \quad$ where $\left(T_{f}-T_{\infty}\right)=T_{0} e^{(\mathrm{A} x / 2 L)}$

$C=C_{\infty}+\left(\mathrm{C}_{f}-C_{\infty}\right) \phi(\zeta)$, where $\left(\mathrm{C}_{f}-C_{\infty}\right)=C_{0} e^{(\mathrm{B} x / 2 L)}, \zeta=z \sqrt{\frac{U_{0}}{2 v L}} e^{(x / 2 L)}$.

where the prime superscripts represent the differentiation concerning $\zeta$.

Using the above transformations, the continuity equation given by Eq (3) is automatically verified, while Eqs. (4) - (7) are reduced to

$$
\begin{gathered}
f^{\prime \prime \prime}+f f^{\prime \prime}-2 f^{\prime 2}+\lambda\left(4 g-2 \beta\left(f g^{\prime}+\zeta f^{\prime \prime} g\right)\right) \\
-\frac{\beta}{2}\left(4 f^{\prime 3}+f^{2} f^{\prime \prime \prime}-\zeta f^{\prime 2} f^{\prime \prime}-6 f f^{\prime} f^{\prime \prime}\right) \\
-\frac{M n}{\left(1+m^{2}\right)}\left(f^{\prime}-\frac{\beta}{2} m g\left(f+\zeta f^{\prime}\right) f^{\prime \prime}\right)=0, \\
g^{\prime \prime}+f g^{\prime}-2 f^{\prime} g+4 \lambda\left(-f^{\prime}+\beta\left(-f^{\prime 2}-g^{2}-\frac{\zeta}{2} g g^{\prime}+\frac{1}{2} f f^{\prime \prime}\right)\right) \\
-\frac{\beta}{2}\left(4 f^{\prime 2} g-\zeta f^{\prime 2} g^{\prime}+f^{2} g^{\prime \prime}-6 f f^{\prime} g^{\prime}\right) \\
-\frac{M n}{\left(1+m^{2}\right)}\left(-\frac{\beta}{2} m f^{\prime}\left(f+\zeta f^{\prime}\right) g^{\prime}-g\right)=0, \\
\theta^{\prime \prime}+\operatorname{Pr}\left(f \theta^{\prime}-A f^{\prime} \theta+N b \theta^{\prime} \phi^{\prime}+N t \theta^{\prime 2}\right)=0, \\
\phi^{\prime \prime}+S c\left(f \phi^{\prime}-\mathrm{B} f^{\prime} \phi\right)+\frac{N t}{N b} \theta^{\prime \prime}=0 .
\end{gathered}
$$

the corresponding BCs Eq. (8) is altered to

$$
\begin{array}{ll}
f(0)=g(0)=0, & f^{\prime}(0)=1+K_{1} f^{\prime \prime}(0), \\
\theta^{\prime}(0)=-B i(1-\theta(0)), & N b \phi^{\prime}(0)+N t \theta^{\prime}(0)=0,
\end{array}
$$$$
f^{\prime}(\infty) \rightarrow 0, \quad g(\infty) \rightarrow 0, \quad \theta(\infty) \rightarrow 0, \quad \phi(\infty) \rightarrow 0
$$

In the above expression, $\lambda$ is the rotation parameter, $\beta$ is the Deborah number, $M n$ is the magnetic parameter, $\operatorname{Pr}$ is Prandtl number, $\mathrm{A}$ is the temperature exponent, $\mathrm{Nb}$ is the Brownian motion parameter, $N t$ is the thermophoresis motion parameter, Sc is the Schmidt number, B is concentration exponent, $K_{1}$ is velocity slip parameter, $B i$ is the Biots number and defined as follow

$\lambda=\frac{\Omega L}{u_{w}}, \quad \beta=\frac{\lambda_{1} u_{w}}{L}, M n=\frac{2 \sigma B_{0}^{2} L^{2}}{\mu \operatorname{Re}_{x}}, \operatorname{Pr}=\frac{v}{\alpha}, N b=\frac{\tau D_{B}\left(C_{f}-C_{\infty}\right)}{v}$,

$N t=\frac{\tau D_{T}\left(T_{f}-T_{\infty}\right)}{v T_{\infty}}, \quad S c=\frac{v}{D_{B}}, \quad K_{1}=k_{1} \sqrt{\frac{U_{0}}{2 v L}} e^{(x / 2 L)}, \quad B i=\frac{h}{k} \sqrt{\frac{2 v L}{U_{0}}}$.

\subsection{Skin-friction, heat and mass transfer coefficients}

The local skin-friction coefficients $\left(C_{f x}, C_{f y}\right)$ and the local Nusselt number $N u_{x}$ are defined formally as

$$
C_{f x}=\frac{v \tau_{w x}}{u_{w}^{2}}, C_{f y}=\frac{v \tau_{w y}}{u_{w}^{2}} \text { and } N u_{x}=-\frac{x q}{\left(T_{f}-T_{\infty}\right)}
$$

where $\tau_{w x}$ and $\tau_{w y}$ are the skin-friction (at wall) along $x$ and $y$ axis, and $q$ is the heat flux from the plate are defined as

$$
\tau_{w x}=\left(\frac{\partial u}{\partial z}\right)_{z=0}, \tau_{w y}=\left(\frac{\partial \mathbf{v}}{\partial z}\right)_{z=0} \text { and } q=\left(\frac{\partial T}{\partial z}\right)_{z=0}
$$

in terms of non-dimensional quantities are obtained as

$$
C_{f x}=\frac{v\left(\frac{\partial u}{\partial z}\right)_{z=0}}{u_{w}^{2}}=\frac{f^{\prime \prime}(0)}{\sqrt{2 \mathrm{Re}_{x}}}, C_{f y}=\frac{v\left(\frac{\partial \mathrm{v}}{\partial z}\right)_{z=0}}{u_{w}^{2}}=\frac{g^{\prime}(0)}{\sqrt{2 \mathrm{Re}_{x}}},
$$

$N u_{x}=\frac{-x\left(\frac{\partial T}{\partial z}\right)_{z=0}}{\left(T_{f}-T_{\infty}\right)}=-\frac{x}{L} \sqrt{\frac{\operatorname{Re}_{x}}{2}} \theta^{\prime}(0)$.

Particularly, for zero nanoparticles mass flux condition, the dimensionless mass flux denoted by the local Sherwood number $S h_{x}$ is identically zero, where $\operatorname{Re}_{x}=u_{w} L / v$ represents the local Reynolds number.

\section{SOLUTION METHODOLOGY BY MEANS OF OHAM}

In this section, we solve Eqs. (10) - (13) with BCs. (14) by a productive semi-analytical algorithm known as Optimal Homotopy Analysis Method (see for details, Liao (2010); Marinca and Herisanu (2015); and Van Gorder (2019)). Consequently, a nonlinear problem is changed into an infinite number of linear sub-problems. In the frame of OHAM, we have an incredible opportunity to pick initial approximation and auxiliary linear operators and are picke as

$$
\begin{aligned}
& f_{0}(\zeta)=\frac{1}{1+K_{1}}\left(1-e^{-\zeta}\right), g_{0}(\zeta)=0, \\
& \theta_{0}(\zeta)=\frac{B i}{1+B i} e^{-\zeta}, \phi_{0}(\zeta)=-\frac{B i}{1+B i}\left(\frac{N t}{N b}\right) e^{-\zeta}, \\
& L_{f}(\zeta)=f^{\prime \prime \prime}-f^{\prime}, L_{g}(\zeta)=g^{\prime \prime}-g, \\
& L_{\theta}(\zeta)=\theta^{\prime \prime}-\theta, L_{\phi}(\zeta)=\phi^{\prime \prime}-\phi .
\end{aligned}
$$

It is worth noting here that the auxiliary linear operators in Eq. (20) satisfy the properties

$$
\left\{\begin{array}{l}
L_{f}\left(A_{1}+A_{2} e^{\zeta}+A_{3} e^{-\zeta}\right)=0, \\
L_{g}\left(A_{4} e^{\zeta}+A_{5} e^{-\zeta}\right)=0, \\
L_{\theta}\left(A_{6} e^{\zeta}+A_{7} e^{-\zeta}\right)=0, \\
L_{\phi}\left(A_{8} e^{\zeta}+A_{9} e^{-\zeta}\right)=0,
\end{array}\right.
$$

where $A_{i}{ }^{\prime} s(i=1-9)$ are arbitrary constants.

In the present procedure, we construct the following zero ${ }^{\text {th }}$-order deformation equations are given by

$$
\begin{aligned}
& (1-q) L_{f}\left[\hat{f}(\zeta ; q)-f_{0}(\zeta)\right]=q H_{f}(\zeta) \hbar_{f} N_{f}[\hat{f}(\zeta ; q), \hat{g}(\zeta ; q)], \\
& (1-q) L_{g}\left[\hat{g}(\zeta ; q)-g_{0}(\zeta)\right]=q H_{g}(\zeta) \hbar_{g} N_{g}[\hat{f}(\zeta ; q), \hat{g}(\zeta ; q)], \\
& (1-q) L_{\theta}\left[\hat{\theta}(\zeta ; q)-\theta_{0}(\zeta)\right]=q H_{\theta}(\zeta) \hbar_{\theta} N_{\theta}[\hat{f}(\zeta ; q), \hat{\theta}(\zeta ; q), \hat{\phi}(\zeta ; q)], \\
& (1-q) L_{\phi}\left[\hat{\phi}(\zeta ; q)-\phi_{0}(\zeta)\right]=q H_{\phi}(\zeta) \hbar_{\phi} N_{\phi}[\hat{f}(\zeta ; q), \hat{\theta}(\zeta ; q), \hat{\phi}(\zeta ; q)],
\end{aligned}
$$

with the following BCs

$$
\begin{aligned}
& \hat{f}(0 ; q)=0, \hat{f}^{\prime}(0 ; q)=1+K_{1} f^{\prime \prime}(0), \\
& \hat{f}^{\prime}(\infty ; q)=0, \hat{g}(0 ; q)=0, \hat{g}(\infty ; q)=0, \\
& \hat{\theta}^{\prime}(0 ; q)=-B i[1-\hat{\theta}(0 ; q)], \hat{\theta}(\infty ; q)=0,
\end{aligned}
$$

$N b \hat{\phi}^{\prime}(0 ; q)+N t \hat{\theta}^{\prime}(0 ; q)=0, \hat{\phi}(\infty ; q)=0$,

where $q \in[0,1]$ is an embedding parameter, $\left(\hbar_{f}, \hbar_{g}, \hbar_{\theta}, \hbar_{\phi}\right)$ are the convergence control parameter and $N_{f}, N_{g}, N_{\theta}, N_{\phi}$ are non-linear operators defined as

$$
\begin{aligned}
N_{f}= & \hat{f}^{\prime \prime \prime}(\zeta, q)+\hat{f}(\zeta, q) \hat{f}^{\prime \prime}(\zeta, q)-2 \hat{f}^{\prime 2}(\zeta, q) \\
& +\lambda\left(4 \hat{g}(\zeta, q)-2 \beta\left(\hat{f}(\zeta, q) \hat{g}^{\prime}(\zeta, q)+\zeta \hat{f}^{\prime \prime}(\zeta, q) \hat{g}(\zeta, q)\right)\right) \\
& -\frac{\beta}{2}\left(\begin{array}{l}
4 \hat{f}^{\prime 3}(\zeta, q)+\hat{f}^{2}(\zeta, q) \hat{f}^{\prime \prime \prime}(\zeta, q) \\
\left.-\xi \hat{f}^{\prime 2}(\zeta, q) \hat{f}^{\prime \prime}(\zeta, q)-6 \hat{f}(\zeta, q) \hat{f}^{\prime}(\zeta, q) \hat{f}^{\prime \prime}(\zeta, q)\right) \\
\end{array}\right. \\
& -\frac{M n}{\left(1+m^{2}\right)}\left(\hat{f}^{\prime}(\zeta, q)-\frac{\beta}{2} m \hat{g}(\zeta, q)\left(\hat{f}(\zeta, q)+\zeta \hat{f}^{\prime}(\zeta, q)\right) \hat{f}^{\prime \prime}(\zeta, q)\right),
\end{aligned}
$$


$N_{g}=\hat{g}^{\prime \prime}(\zeta, q)+\hat{f}(\zeta, q) \hat{g}^{\prime}(\zeta, q)-2 \hat{f}^{\prime}(\zeta, q) \hat{g}(\zeta, q)$

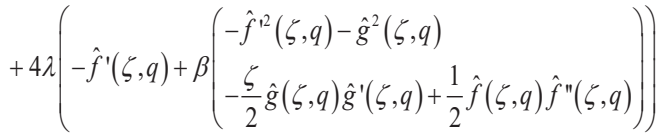

$$
\begin{aligned}
& -\frac{\beta}{2}\left(\begin{array}{l}
4 \hat{f}^{\prime 2}(\zeta, q) \hat{g}(\zeta, q)-\xi \hat{f}^{\prime 2}(\zeta, q) \hat{g}^{\prime}(\zeta, q) \\
+\hat{f}^{2}(\zeta, q) \hat{g}^{\prime \prime}(\zeta, q)-6 \hat{f}(\zeta, q) \hat{f}^{\prime}(\zeta, q) \hat{g}^{\prime}(\zeta, q)
\end{array}\right) \\
& -\frac{M n}{\left(1+m^{2}\right)}\left(-\frac{\beta}{2} m \hat{f}^{\prime}(\zeta, q)\left(\hat{f}(\zeta, q)+\zeta \hat{f}^{\prime}(\zeta, q)\right) \hat{g}^{\prime}(\zeta, q)-\hat{g}(\zeta, q)\right), \\
& N_{\theta}=\hat{\theta}^{\prime \prime}(\zeta, q)+\operatorname{Pr}\left(\begin{array}{l}
\hat{f}(\zeta, q) \hat{\theta}^{\prime}(\zeta, q)-\mathrm{A} \hat{f}^{\prime}(\zeta, q) \hat{\theta}(\zeta, q) \\
+N b \hat{\theta}^{\prime}(\zeta, q) \hat{\phi}^{\prime}(\zeta, q)+N t \hat{\theta}^{2}(\zeta, q)
\end{array}\right), \\
& N_{\phi}=\hat{\phi}^{\prime \prime}(\zeta, q)+S c\left(\begin{array}{l}
\hat{f}(\zeta, q) \hat{\phi}^{\prime}(\zeta, q) \\
-B \hat{f}^{\prime}(\zeta, q) \hat{\phi}(\zeta, q)
\end{array}\right)+\frac{N t}{N b} \hat{\theta}^{\prime \prime}(\zeta, q) .
\end{aligned}
$$

Methodologically, we choose the auxiliary function as $H_{f}(\zeta)=H_{g}(\zeta)=H_{\theta}(\zeta)=H_{\phi}(\zeta)=e^{-\zeta}$

Hence, by setting $q=0$ and $q=1$, in Eq. (22) then we get the solutions as follows

$\int \hat{f}(\zeta ; 0)=\hat{f}_{0}(\zeta), \hat{g}(\zeta ; 0)=\hat{g}_{0}(\zeta), \hat{\theta}(\zeta ; 0)=\hat{\theta}_{0}(\zeta), \hat{\phi}(\zeta ; 0)=\hat{\phi}_{0}(\zeta)$, $\hat{f}(\zeta ; 1)=\hat{f}(\zeta), \hat{g}(\zeta ; 1)=\hat{g}(\zeta), \hat{\theta}(\zeta ; 1)=\hat{\theta}(\zeta), \hat{\phi}(\zeta ; 1)=\hat{\phi}(\zeta)$.

From Eq. (28), it is clear that $\hat{f}(\zeta, q), \hat{g}(\zeta, q), \hat{\theta}(\zeta, q)$ and $\hat{\phi}(\zeta, q)$ vary from the initial guesses $f_{0}(\zeta), g_{0}(\zeta), \theta_{0}(\zeta)$ and $\phi_{0}(\zeta)$ to the final solutions $f(\zeta), g(\zeta), \theta(\zeta)$ and $\phi(\zeta)$ of Eqs. (10) - (13) with BCs. (14), when the parameter $q$ oscillates between 0 to 1 . Moreover, the sought solutions $\hat{f}(\zeta, q), \hat{g}(\zeta, q), \hat{\theta}(\zeta, q)$ and $\hat{\phi}(\zeta, q)$ can be approximated accurately for the variable $q$ by higherorder expansions with the help of Taylor's series as follows

$$
\left\{\begin{array}{l}
\hat{f}(\zeta ; q)=f_{0}(\zeta)+\sum_{p=1}^{\infty} f_{p}(\zeta) q^{p}, \quad f_{p}(\zeta)=\left.\frac{1}{\mathrm{p} !} \frac{d^{p} f(\zeta ; q)}{d \zeta^{p}}\right|_{q=0} \\
\hat{g}(\zeta ; q)=g_{0}(\zeta)+\sum_{p=1}^{\infty} g_{p}(\zeta) q^{p}, g_{p}(\zeta)=\left.\frac{1}{\mathrm{p} !} \frac{d^{p} g(\zeta ; q)}{d \zeta^{p}}\right|_{q=0} \\
\hat{\theta}(\zeta ; q)=\theta_{0}(\zeta)+\sum_{p=1}^{\infty} \theta_{p}(\zeta) q^{p}, \theta_{p}(\zeta)=\left.\frac{1}{\mathrm{p} !} \frac{d^{p} \theta(\zeta ; q)}{d \zeta^{p}}\right|_{q=0} \\
\hat{\phi}(\zeta ; q)=\phi_{0}(\zeta)+\sum_{p=1}^{\infty} \phi_{p}(\zeta) q^{p}, \quad \phi_{p}(\zeta)=\left.\frac{1}{\mathrm{p} !} \frac{d^{p} \phi(\zeta ; q)}{d \zeta^{p}}\right|_{q=0}
\end{array}\right.
$$

where the convergence of the above series strongly depends upon $\hbar_{f}, \hbar_{g}, \hbar_{\theta}$ and $\hbar_{\phi}$. Considering that the control parameter $\left(\hbar_{f}, \hbar_{g}, \hbar_{\theta}, \hbar_{\phi}\right)$ are chosen in such a manner that Eq. (22) converges at $q=1$. Then, we have

$$
\left\{\begin{array}{l}
f(\zeta)=f_{0}(\zeta)+\sum_{p=1}^{\infty} f_{p}(\zeta), g(\zeta)=g_{0}(\zeta)+\sum_{p=1}^{\infty} g_{p}(\zeta) \\
\theta(\zeta)=\theta_{0}(\zeta)+\sum_{p=1}^{\infty} \theta_{p}(\zeta), \phi(\zeta)=\phi_{0}(\zeta)+\sum_{p=1}^{\infty} \phi_{p}(\zeta)
\end{array}\right.
$$

The $\mathrm{p}^{\text {th }}$ - order deformation equations and their corresponding BCs are

$$
\begin{aligned}
& \left\{\begin{array}{l}
L_{f}\left[f_{p}(\zeta)-\chi_{p} f_{p-1}(\zeta)\right]=\hbar_{f} R_{p}^{f}(\zeta), L_{g}\left[g_{p}(\zeta)-\chi_{p} g_{p-1}(\zeta)\right]=\hbar_{g} R_{p}^{g}(\zeta) \\
L_{\theta}\left[\theta_{p}(\zeta)-\chi_{p} \theta_{p-1}(\zeta)\right]=\hbar_{\theta} R_{p}^{\theta}(\zeta), L_{\phi}\left[\phi_{p}(\zeta)-\chi_{p} \phi_{p-1}(\zeta)\right]=\hbar_{\phi} R_{p}^{\phi}(\zeta)
\end{array}\right. \\
& \left\{\begin{array}{l}
f_{p}(0)=0, f^{\prime}{ }_{p}(0)-K_{1} f_{p}{ }^{\prime \prime}(0)=0, f^{\prime}{ }_{p}(\infty)=0 \\
g_{p}(0)=0, g_{p}(\infty)=0 \\
\theta^{\prime}{ }_{p}(0)=-B i\left(1-\theta_{p}(0)\right), \theta_{p}(\infty)=0 \\
N b \phi_{p}{ }^{\prime}(0)+N t \theta^{\prime}{ }_{p}(0)=0, \phi_{p}(\infty)=0
\end{array}\right.
\end{aligned}
$$

Where

$$
\begin{aligned}
& R_{p}^{f}=f^{\prime{ }^{\prime \prime}}{ }_{p-1}(\zeta)+\sum_{k=0}^{p-1} f_{p-1-k} f^{\prime \prime}{ }_{k}-2 \sum_{k=0}^{p-1} f^{\prime}{ }_{p-1-k} f^{\prime}{ }_{k} \\
& +\lambda\left(4 g_{m-1-k}-2 \beta \sum_{k=0}^{p-1} f_{p-1-k} \mathrm{~g}_{k}^{\prime}-2 \beta \zeta \sum_{k=0}^{p-1} \mathrm{~g}_{p-1-k} f^{\prime \prime}{ }_{k}\right) \\
& -\frac{\beta}{2}\left(\begin{array}{l}
4 \sum_{k=0}^{p-1}\left(f_{p-1-k}^{\prime} f_{k-l}^{\prime}\right) f^{\prime}{ }_{l}+\sum_{k=0}^{p-1}\left(f_{p-1-k} f_{k-l}\right) f^{\prime{ }^{\prime}{ }_{l}} \\
-\zeta \sum_{k=0}^{p-1}\left(f_{p-1-k}^{\prime} f_{k-1}^{\prime}\right) f^{\prime \prime}{ }_{l}-6 \sum_{k=0}^{p-1}\left(f_{p-1-k} f^{\prime}{ }_{k-1}\right) f^{\prime \prime}{ }_{l}
\end{array}\right) \\
& -\frac{M n}{\left(1+m^{2}\right)}\left(f_{p-k-l}^{\prime}-\frac{\beta}{2} m g_{p-k-1}\left(\sum_{k=0}^{p-1} f_{p-1-k} f^{\prime \prime}{ }_{k}+\zeta \sum_{k=0}^{p-1} f_{p-1-k}^{\prime} f_{k}^{\prime \prime}\right)\right) \\
& R_{p}^{g}=g_{p-1}^{\prime \prime}(\zeta)+\sum_{k=0}^{p-1} f_{p-1-k} g_{k}^{\prime}-2 \sum_{k=0}^{p-1} f^{\prime}{ }_{p-1-k} g_{k}
\end{aligned}
$$

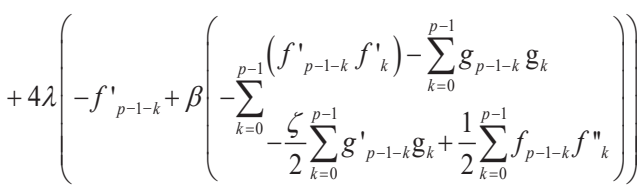

$$
\begin{aligned}
& -\frac{\beta}{2}\left(\begin{array}{l}
4 \sum_{k=0}^{p-1}\left(f_{p-1-k}^{\prime} f_{k-l}^{\prime}\right) g_{l}-\zeta \sum_{k=0}^{p-1}\left(f_{p-1-k}^{\prime} f_{k-l}^{\prime}\right) \mathrm{g}_{l}^{\prime} \\
+\sum_{k=0}^{p-1}\left(f_{p-1-k} f_{k-1}\right) g^{\prime \prime}{ }_{l}-6 \sum_{k=0}^{p-1}\left(f_{p-1-k} f_{k-1}^{\prime}\right) \mathrm{g}_{l}^{\prime}
\end{array}\right) \\
& -\frac{M n}{\left(1+m^{2}\right)}\left(-\frac{\beta}{2} m f^{\prime}{ }_{p-k-1}\left(\sum_{k=0}^{p-1} f_{p-1-k} \mathrm{~g}_{k}^{\prime}+\zeta \sum_{k=0}^{p-1}{f^{\prime}}_{p-1-k} \mathrm{~g}_{k}^{\prime}\right)-g_{p-k-1}\right) \\
& R_{p}^{\theta}=\theta_{p-1}^{\prime \prime}(\zeta)+\operatorname{Pr} \sum_{k=0}^{p-1}\left(\theta_{p-1-k}^{\prime} f_{k}-A \theta_{p-1-k} f^{\prime}{ }_{k}\right) \\
& +N b \sum_{k=0}^{p-1} \theta_{p-1-k}^{\prime} \phi_{k}^{\prime}+N t \sum_{k=0}^{p-1} \theta^{\prime}{ }_{p-1-k} \theta^{\prime}{ }_{k} \\
& R_{p}^{\phi}=\phi^{\prime \prime}{ }_{p-1}(\zeta)+S c \sum_{k=0}^{p-1}\left(\phi_{p-1-k}^{\prime} f_{k}-B \phi_{p-1-k} f^{\prime}{ }_{k}\right)+\frac{N t}{N b} \theta_{p-1}^{\prime \prime}(\zeta) \\
& \chi_{p}=\left\{\begin{array}{l}
0, p \leq 1 \\
1, p>1
\end{array}\right.
\end{aligned}
$$

The general solutions of the $\mathrm{p}^{\text {th }}$-order deformation equations are given by

$$
\left\{\begin{array}{l}
f_{p}(\zeta)=f_{p}^{*}(\zeta)+A_{1}+A_{2} e^{\zeta}+A_{3} e^{-\zeta} \\
g_{p}(\zeta)=g_{p}^{*}(\zeta)+A_{4}+A_{5} e^{\zeta}+A_{6} e^{-\zeta} \\
\theta_{p}(\zeta)=\theta_{p}^{*}(\zeta)+A_{7} e^{\zeta}+A_{8} e^{-\zeta} \\
\phi_{p}(\zeta)=\phi_{p}^{*}(\zeta)+A_{9} e^{\zeta}+A_{10} e^{-\zeta}
\end{array}\right.
$$

in which $\left(f_{p}^{*}(\zeta), g_{p}^{*}(\zeta), \theta_{p}^{*}(\zeta), \phi_{p}^{*}(\zeta)\right)$ denote the special solutions.

The expressions of exact residual errors are written as follows

$$
\left\{\begin{array}{l}
\widehat{E}_{p}^{f}(\hbar)=\int_{0}^{1}\left(N_{f}\left[\sum_{k=0}^{p} f_{k}(\zeta), \sum_{k=0}^{p} g_{k}(\zeta)\right]\right)^{2} d \zeta \\
\widehat{E}_{p}^{g}(\hbar)=\int_{0}^{1}\left(N_{g}\left[\sum_{k=0}^{p} f_{k}(\zeta), \sum_{k=0}^{p} g_{k}(\zeta)\right]\right)^{2} d \zeta \\
\hat{E}_{p}^{\theta}(\hbar)=\int_{0}^{1}\left(N_{\theta}\left[\sum_{k=0}^{p} f_{k}(\zeta), \sum_{k=0}^{p} \theta_{k}(\zeta), \sum_{k=0}^{p} \phi_{k}(\zeta)\right]\right)^{2} d \zeta \\
\widehat{E}_{p}^{\phi}(\hbar)=\int_{0}^{1}\left(N_{\phi}\left[\sum_{k=0}^{p} f_{k}(\zeta), \sum_{k=0}^{p} \theta_{k}(\zeta), \sum_{k=0}^{p} \phi_{k}(\zeta)\right]\right)^{2} d \zeta
\end{array}\right.
$$

In practice the evaluation of the residual errors are $\left(\hat{E}_{p}^{f}(\hbar), \hat{E}_{p}^{g}(\hbar), \hat{E}_{p}^{\theta}(\hbar), \hat{E}_{p}^{\phi}(\hbar)\right)$ consumed a large amount of time. So, instead of computing the exact residual errors, it is feasible to handle the accuracy of the problem using the average residual errors defined by 


$$
\left\{\begin{array}{l}
\hat{E}_{p}^{f}(\hbar)=\frac{1}{P+1} \sum_{k=0}^{P}\left(N_{f}\left(\sum_{n=0}^{p} f(\zeta), \sum_{n=0}^{p} g(\zeta)\right)_{\zeta=n \delta \zeta}\right)^{2} \\
\hat{E}_{p}^{g}(\hbar)=\frac{1}{P+1} \sum_{k=0}^{P}\left(N_{g}\left(\sum_{n=0}^{p} f(\zeta), \sum_{n=0}^{p} g(\zeta)\right)_{\zeta=n \delta \zeta}\right)^{2} \\
\hat{E}_{p}^{\theta}(\hbar)=\frac{1}{P+1} \sum_{k=0}^{P}\left(N_{\theta}\left(\sum_{n=0}^{p} f(\zeta), \sum_{n=0}^{p} \theta(\zeta), \sum_{n=0}^{p} \phi(\zeta)\right)_{\zeta=n \delta \zeta}\right)^{2} \\
\hat{E}_{p}^{\phi}(\hbar)=\frac{1}{P+1} \sum_{k=0}^{P}\left(N_{\phi}\left(\sum_{n=0}^{p} f(\zeta), \sum_{n=0}^{p} \theta(\zeta), \sum_{n=0}^{p} \phi(\zeta)\right)_{\zeta=n \delta \zeta}\right. \\
\hat{E}_{p}^{t}=\hat{E}_{p}^{f}+\hat{E}_{p}^{g}+\hat{E}_{p}^{\theta}+\hat{E}_{p}^{\phi}
\end{array}\right.
$$

where $\hat{E}_{p}^{t}$ represents the total squared residual error, $\zeta=n \delta \zeta=k / \mathrm{P}, k=0,1,2 . ., \mathrm{P}$.

Now we minimize the error function $\hat{E}_{p}^{f}(\hbar), \hat{E}_{p}^{g}(\hbar), \hat{E}_{p}^{\theta}(\hbar)$ and $\hat{E}_{p}^{\phi}(\hbar)$ and record the optimal values of $\left(\hbar_{f}, \hbar_{g}, \hbar_{\theta}, \hbar_{\phi}\right)$. For the required order approximation, the optimal values of $\left(\hbar_{f}, \hbar_{g}, \hbar_{\theta}, \hbar_{\phi}\right)$ corresponding to the functions $(f, g, \theta, \phi)$ are obtained by utilizing the following mathematical restrictions

$\frac{d \hat{E}_{p}^{f}(\hbar)}{d \hbar}=0, \frac{d \hat{E}_{p}^{g}(\hbar)}{d \hbar}=0, \frac{d \hat{E}_{p}^{\theta}(\hbar)}{d \hbar}=0, \frac{d \hat{E}_{p}^{\phi}(\hbar)}{d \hbar}=0$

The convergent series solutions correspond to

$$
\lim _{p \rightarrow \infty} \hat{E}_{p}^{f}(\hbar)=0, \lim _{p \rightarrow \infty} \hat{E}_{p}^{g}(\hbar)=0, \lim _{p \rightarrow \infty} \hat{E}_{p}^{\theta}(\hbar)=0, \lim _{p \rightarrow \infty} \hat{E}_{p}^{\phi}(\hbar)=0
$$

Table 1 and 2 provide the results obtained for the individual average squared residual error and total residual error by considering $\left(\hbar_{f}, \hbar_{g}, \hbar_{\theta}, \hbar_{\phi}\right)=-(1.18152,1.29527,1.30537,1.46363)$ as the optimal values which have been analyzed by minimizing the averaged residual error and total residual error at $12^{\text {th }}$-order approximation. It can be observed that the averaged squared residual error, and total residual errors are consistently reduced as increases the order of approximations. Further, the average squared residual error and total residual error of each governing equations are found in the diminishing function of the order of approximation, as shown in Fig. 2(a-b).

Table 1 The individual average squared residual error as a function of the number of iterations and CPU time is also listed. Physical parameters are $\operatorname{Pr}=1.09, S c=m=1, N b=M n=B i=0.5, N t=\lambda=0.1$,

$\mathrm{A}=\mathrm{B}=\beta=K_{1}=0.2$, fixed and $\mathrm{CPU}$ time is also listed. We obtain the optimal values of convergence control parameters are $\hbar_{f}=-1.18152, \hbar_{g}=-1.29527, \hbar_{\theta}=-1.30537, \hbar_{\phi}=-1.46363$.

\begin{tabular}{cccccc}
\hline$p$ & $\hat{E}_{p}^{f}$ & $\hat{E}_{p}^{g}$ & $\hat{E}_{p}^{\theta}$ & $\hat{E}_{p}^{\phi}$ & CPU time \\
\hline 2 & $6.09 \times 10^{-4}$ & $2.86 \times 10^{-3}$ & $2.57 \times 10^{-5}$ & $3.53 \times 10^{-6}$ & $3.51 \mathrm{~s}$ \\
4 & $3.59 \times 10^{-5}$ & $3.89 \times 10^{-4}$ & $8.28 \times 10^{-7}$ & $6.19 \times 10^{-7}$ & $34.59 \mathrm{~s}$ \\
6 & $4.96 \times 10^{-6}$ & $9.74 \times 10^{-5}$ & $4.40 \times 10^{-9}$ & $8.93 \times 10^{-8}$ & $263.18 \mathrm{~s}$ \\
8 & $1.17 \times 10^{-6}$ & $3.55 \times 10^{-5}$ & $1.31 \times 10^{-9}$ & $1.69 \times 10^{-8}$ & $1261.57 \mathrm{~s}$ \\
10 & $4.16 \times 10^{-7}$ & $1.67 \times 10^{-5}$ & $1.11 \times 10^{-9}$ & $5.52 \times 10^{-9}$ & $4898.92 \mathrm{~s}$ \\
12 & $1.97 \times 10^{-7}$ & $9.29 \times 10^{-6}$ & $3.21 \times 0^{-10}$ & $2.27 \times 10^{-9}$ & $24101.19 \mathrm{~s}$ \\
\hline
\end{tabular}

Table 2 Total averaged squared residual error with no. of iteration and CPU time(s). Physical parameters are $\operatorname{Pr}=1.09, S c=m=1$, $N b=M n=B i=0.5, N t=\lambda=0.1, \mathrm{~A}=\mathrm{B}=\beta=K_{1}=0.2$, fixed and recorded as CPU time (in sec).

\begin{tabular}{ccccccc}
\hline$p$ & $-\bar{h}_{p}^{f}$ & $-\bar{h}_{p}^{g}$ & $\bar{h}_{p}^{\theta}$ & $\bar{h}_{p}^{\phi}$ & $\bar{E}_{p}^{t}$ & CPU time \\
\hline 2 & 0.96748 & 1.17805 & 1.21602 & 1.39283 & $1.05 \times 10^{-2}$ & $1.81 \mathrm{~s}$ \\
4 & 1.05049 & 1.24464 & 1.20643 & 1.49248 & $9.36 \times 10^{-3}$ & $7.86 \mathrm{~s}$ \\
6 & 1.10042 & 1.26462 & 1.17431 & 1.52095 & $1.75 \times 10^{-4}$ & $15.46 \mathrm{~s}$ \\
8 & 1.13393 & 1.27572 & 0.95993 & 1.50951 & $3.38 \times 10^{-5}$ & $170.42 \mathrm{~s}$ \\
10 & 1.15996 & 1.28557 & 0.81760 & 1.48142 & $2.52 \times 10^{-5}$ & $318.52 \mathrm{~s}$ \\
12 & 1.18152 & 1.29527 & 0.71537 & 1.46363 & $1.39 \times 10^{-5}$ & $526.91 \mathrm{~s}$ \\
\hline
\end{tabular}

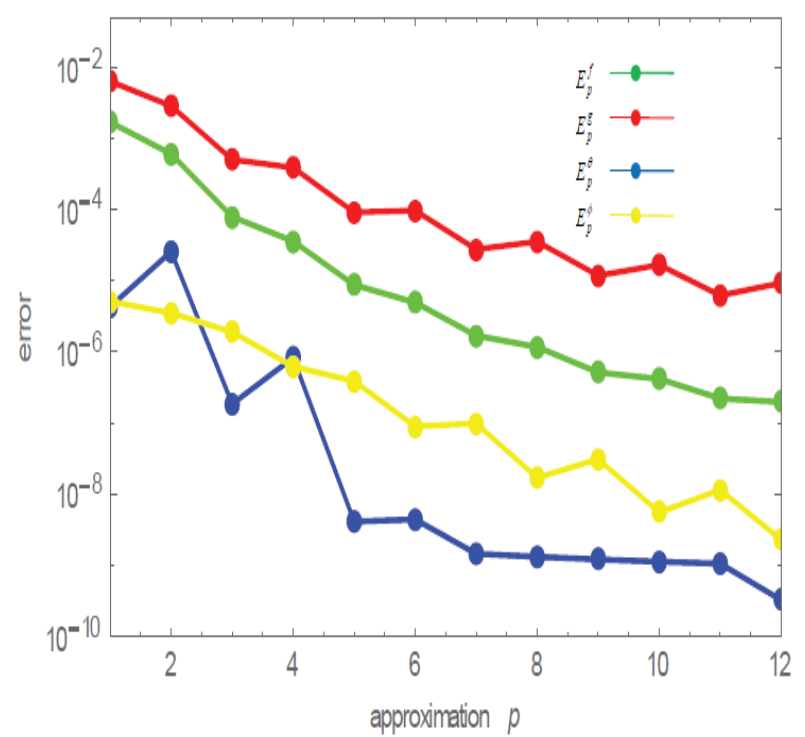

Fig. 2(a) Residual error vs Order of approximation

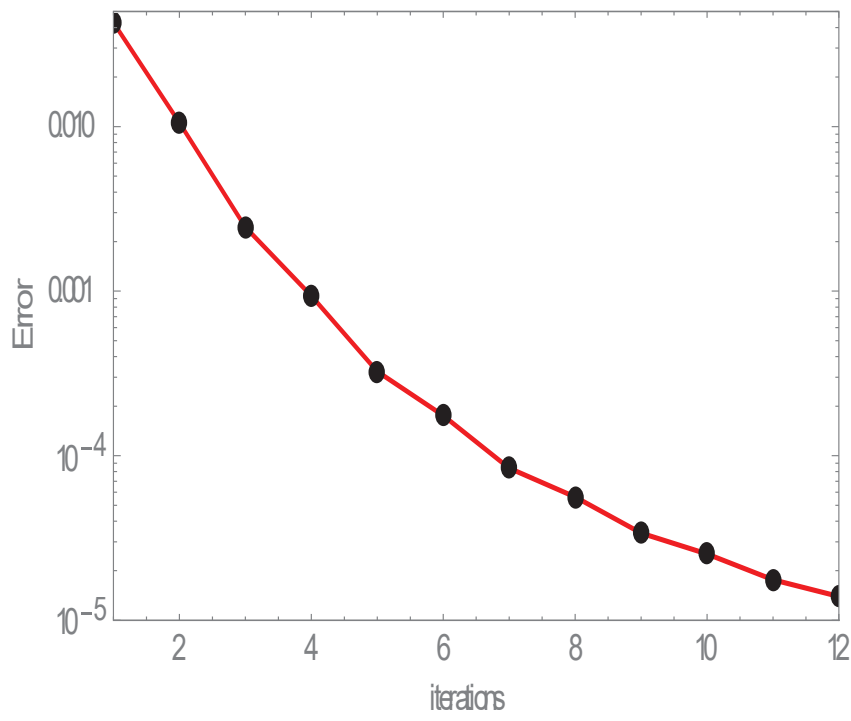

Fig. 2(b) Total residual error vs. no. of iteration $p$.

\section{MODEL VALIDATION}

Here, we present the exact solutions in certain special cases. These solutions have much importance due to they provide as a baseline for the comparison with the obtained results in the literature through the numerical solutions. In the absence of somenon-dimensional parameters, (i.e., $\left.\left(\beta, M n, m, K_{1}, A, B\right)=(0,0,0,0,0,0)\right)$, Eqs. (10) - (13) and BCs. (14) are reduced to those treated by Mustafa et al. (2016), which are

$$
\begin{aligned}
& f^{\prime \prime}+f f f^{\prime \prime}-2 f^{\prime 2}+4 \lambda g=0, \\
& g^{\prime \prime}+f g^{\prime}-2 f^{\prime} \mathrm{g}-4 \lambda f^{\prime}=0, \\
& \theta^{\prime \prime}+\operatorname{Pr}\left(f \theta^{\prime}+N b \theta^{\prime} \phi^{\prime}+N t \theta^{\prime 2}\right)=0, \\
& \phi^{\prime \prime}+S c f \phi^{\prime}+\frac{N t}{N b} \theta^{\prime \prime}=0,
\end{aligned}
$$

with boundary conditions are

$$
\begin{aligned}
& f(0)=g(0)=0, f^{\prime}(0)=1, \theta^{\prime}(0)=-B i(1-\theta(0)), N b \phi^{\prime}(0)+N t \theta^{\prime}(0)=0 \\
& f^{\prime}(\infty) \rightarrow 0, \quad g(\infty) \rightarrow 0, \quad \theta(\infty) \rightarrow 0, \quad \phi(\infty) \rightarrow 0
\end{aligned}
$$

To authenticate and validate the exactness of the proposed OHAM procedure, the present outcomes are compared with those reported by 
Hayat et al. (2017) for some special cases. The correctness found to be in superior agreement (see Table 3 ).

Table 3 Comparison results of local Nusselt number and local Sherwood number for various values of $\beta$ and $\lambda$ when $M n=m=K_{1}=B i=0$.

\begin{tabular}{lccccc}
\hline & & \multicolumn{2}{c}{ Hayat et al. } & \multirow{2}{2}{$(2017)$} & \multicolumn{2}{c}{$\begin{array}{c}\text { Present Results } \\
\text { By OHAM }\end{array}$} \\
\cline { 3 - 6 }$\beta$ & $\lambda$ & $-\theta^{\prime}(0)$ & $-\phi^{\prime}(0)$ & $-\theta^{\prime}(0)$ & $-\phi^{\prime}(0)$ \\
\hline 0.0 & & 0.5323 & 0.5040 & 0.532478 & 0.504141 \\
0.1 & 0.1 & 0.5239 & 0.4943 & 0.524068 & 0.494511 \\
0.2 & & 0.5172 & 0.4858 & 0.519813 & 0.490602 \\
\hline & 0.0 & 0.5358 & 0.5107 & 0.536286 & 0.512786 \\
0.1 & 0.1 & 0.5239 & 0.4943 & 0.525566 & 0.498645 \\
& 0.2 & 0.4988 & 0.4609 & 0.500721 & 0.465365 \\
\hline
\end{tabular}

\section{RESULTS AND DISCUSSION}

The obtained numerical results by means of solving the transformed Eqs. (10) - (13) which are coupled and non-linear are treated with OHAM technique. Thus obtained are analyzed with the help of figures and tables. The results for the horizontal velocity profiles $f^{\prime}(\zeta)$, transverse velocity profiles $\mathrm{g}(\zeta)$, temperature profiles $\theta(\zeta)$ and concentration profiles $\phi(\zeta)$ are presented for various pertinent flow parameters such as rotation parameter $(\lambda)$, Deborah number $(\beta)$, magnetic parameter $(M n)$, hall current parameter $(m)$, Prandtl number $(\mathrm{Pr})$, temperature exponent (A), Brownian motion parameter $(\mathrm{Nb})$, thermophoresis parameter $(\mathrm{Nt})$, Schmidt number $(S c)$, concentration exponent $(\mathrm{B})$, velocity slip parameter $\left(K_{1}\right)$ and Biots number $(B i)$ in Figs. 3 - 7. The computed numerical values for the skin-friction coefficient $f^{\prime \prime}(0)$ and $g^{\prime}(0)$, the local Nusselt number $\theta^{\prime}(0)$, and the local Sherwood number $\phi^{\prime}(0)$ are tabulated in Table 4.

Figure 3(a) through 3(d) elucidates the graphical representation of the profiles $f^{\prime}(\zeta), g(\zeta), \theta(\zeta)$ and $\phi(\zeta)$ for different values of $\lambda$ and $\beta$. Figure 3(a) depicts that the suppressed horizontal velocity field $f^{\prime}(\zeta)$ for the rising values of both $\lambda$ and $\beta$. The rotation parameter $\lambda$ decreases the velocity profile $g(\zeta)$ within the range $0<\zeta<2$ and then enhances gradually in the range $2<\zeta<7$.

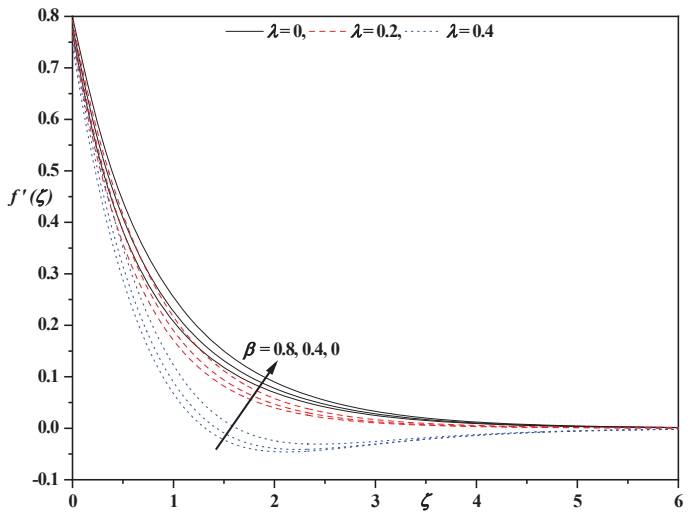

Fig. 3(a) Horizontal velocity profiles for different values of $\lambda$ and $\beta$ with $\operatorname{Pr}=1.09, S c=m=1, N b=B i=M n=0.5$, $N t=0.1, \mathrm{~A}=\mathrm{B}=\mathrm{K}_{1}=0.2$.
Physically, this dual behavior of the fluid flow is due to the larger values of the rotation parameter $\lambda$ correspond to a higher rotation for the angular velocity $\Omega$, because the term $\lambda$ is defined as $\lambda=\Omega L / u_{w}$.

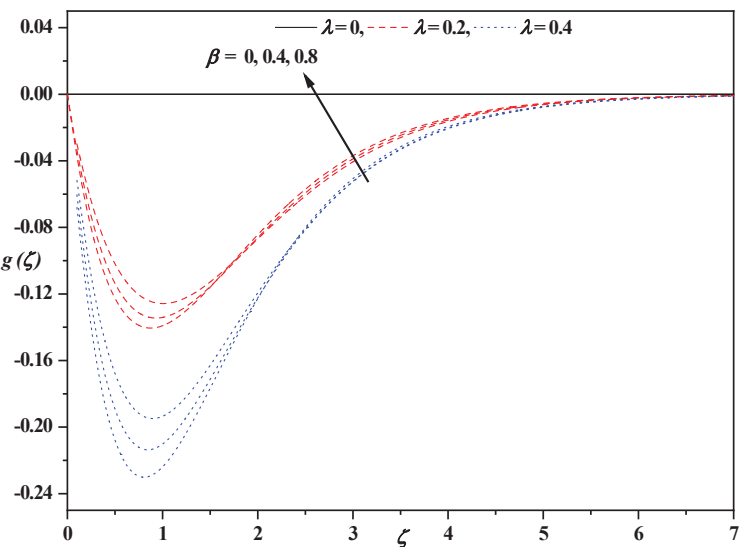

Fig. 3(b) Transverse velocity profiles for different values of $\lambda$ and $\beta$ with $\operatorname{Pr}=1.09, S c=m=1, N b=B i=M n=0.5$, $N t=0.1, \mathrm{~A}=\mathrm{B}=\mathrm{K}_{1}=0.2$.

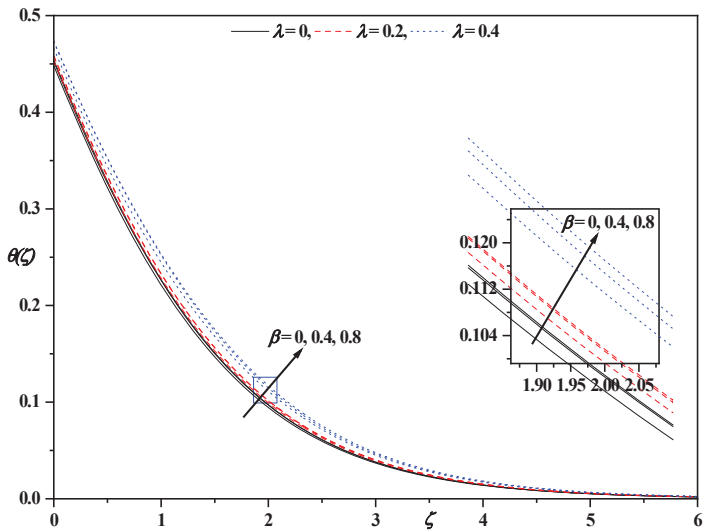

Fig. 3(c) Temperature profiles for different values of $\lambda$ and $\beta$ with $\operatorname{Pr}=1.09, S c=m=1, N b=B i=M n=0.5$, $N t=0.1, \mathrm{~A}=\mathrm{B}=\mathrm{K}_{1}=0.2$.

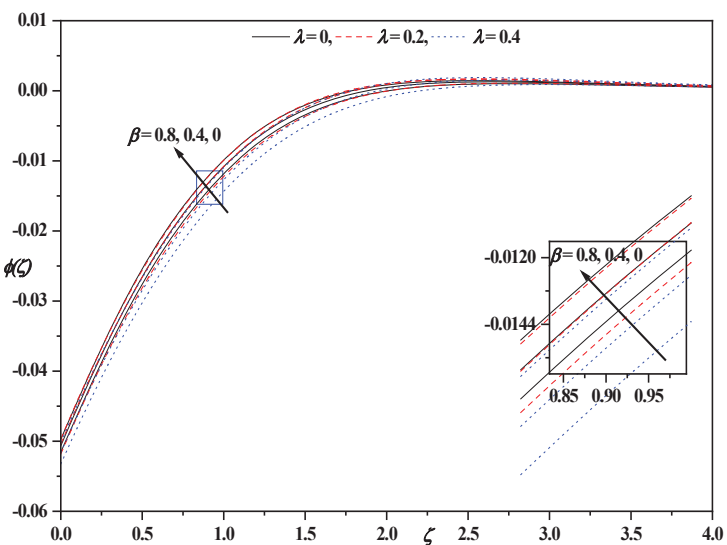

Fig. 3(d) Concentration profiles for different values of $\lambda$ and $\beta$ with $\operatorname{Pr}=1.09, S c=m=1, N b=B i=M n=0.5$, $N t=0.1, \mathrm{~A}=\mathrm{B}=\mathrm{K}_{1}=0.2$. 
Further, the smaller Deborah number $\beta$ gives a viscous effect compared to the elastic effect, whereas the larger $\beta$ exhibit in the elastically solid material in nature. With reference to $\theta(\zeta)$, both $\lambda$ and $\beta$ increases the temperature field, which is recorded in Fig. 3(c). The concentration profile exhibits the decreasing trend for $\lambda$ and $\beta$ (see Fig. 3(d)). Figure 4(a) to 4(d) illustrates the impact of the magnetic parameter $M n$ (presence and absence) and the hall current parameter $m$ on $f^{\prime}(\zeta), g(\zeta), \theta(\zeta)$ and $\phi(\zeta)$. It is observed that the rising values of $m$ increases $f^{\prime}(\zeta), g(\zeta)$ and $\phi(\zeta)$ and decreases $\theta(\zeta)$. This phenomenon is obtained due to the effective conductivity $\left[\sigma /\left(1+m^{2}\right)\right]$, which decreases with increasing values of $m$. Hence, it reduces the applied magnetic field, and consequently increases $f^{\prime}(\zeta)$.

Further, for a larger value of hall current parameter $m$, the term $\left[1 /\left(1+m^{2}\right)\right]$ becomes smaller and smaller, and the resistive force of the magnetic field is diminished. Besides this, the magnetic parameter $M n$ reduces the fluid velocity, concentration profile in the boundary region and enhances the temperature field. The reason behind this development is the opposing force known as the Lorentz force, and this force tends to slow down the fluid flow.

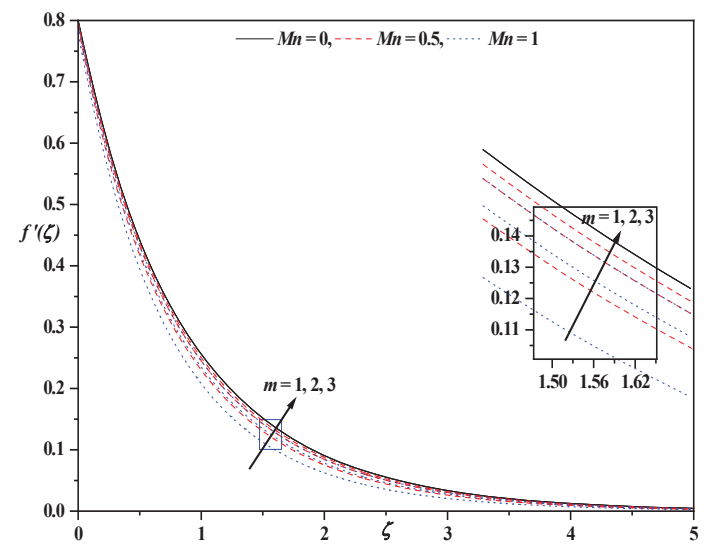

Fig. 4(a) Horizontal velocity profiles for different values of $M n$ and $m$ with $\operatorname{Pr}=1.09, S c=1, N b=B i=0.5, N t=\lambda=0.1$, $\mathrm{A}=\mathrm{B}=\mathrm{K}_{1}=\beta=0.2$.

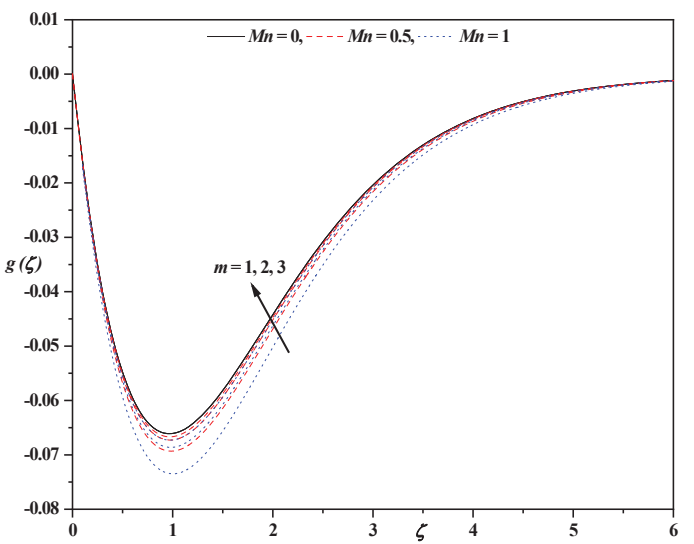

Fig. 4(b) Transverse velocity profiles for different values of $M n$ and $m$ with $\operatorname{Pr}=1.09, S c=1, N b=B i=0.5, N t=\lambda=0.1$, $\mathrm{A}=\mathrm{B}=\mathrm{K}_{1}=\beta=0.2$.

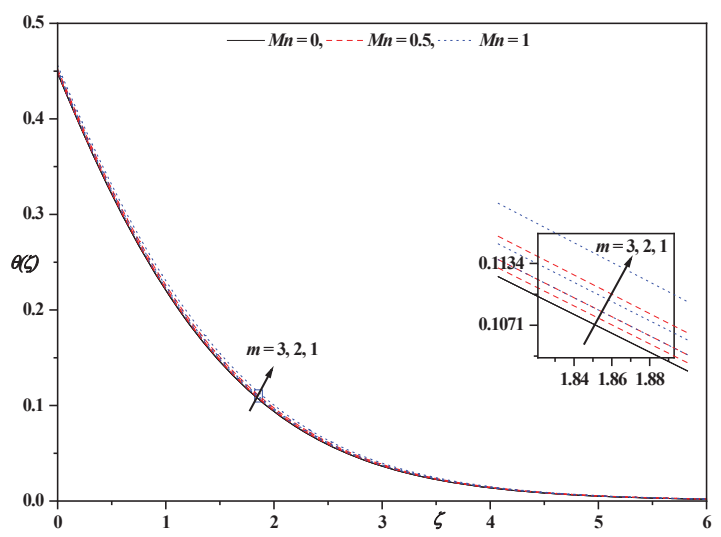

Fig. 4(c) Temperature profiles for different values of $M n$ and $m$ with $\operatorname{Pr}=1.09, S c=1, N b=B i=0.5, N t=\lambda=0.1$, $\mathrm{A}=\mathrm{B}=\mathrm{K}_{1}=\beta=0.2$.

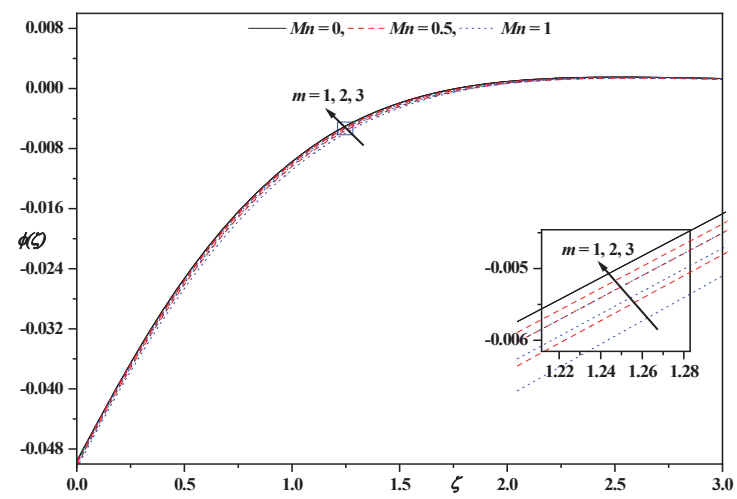

Fig. 4(d) Concentration profiles for different values of $M n$ and $m$ with $\operatorname{Pr}=1.09, S c=1, N b=B i=0.5, N t=\lambda=0.1$, $\mathrm{A}=\mathrm{B}=\mathrm{K}_{1}=\beta=0.2$.

The influence of the magnetic parameter $M n$ (in the presence /absence) and velocity slip $K_{1}$ on $f^{\prime}(\zeta)$ is elucidated in Fig. 5. The velocity profile $f^{\prime}(\zeta)$ decreases for varying values of $K_{1}$. Physically, the higher the value of $K_{1}$ reduces the kinematic viscosity $\mathcal{V}$. This nature of the profile is attributed to the fact $K_{1}=k_{1} \sqrt{U_{0} / 2 v L} e^{(x / 2 L)}$.

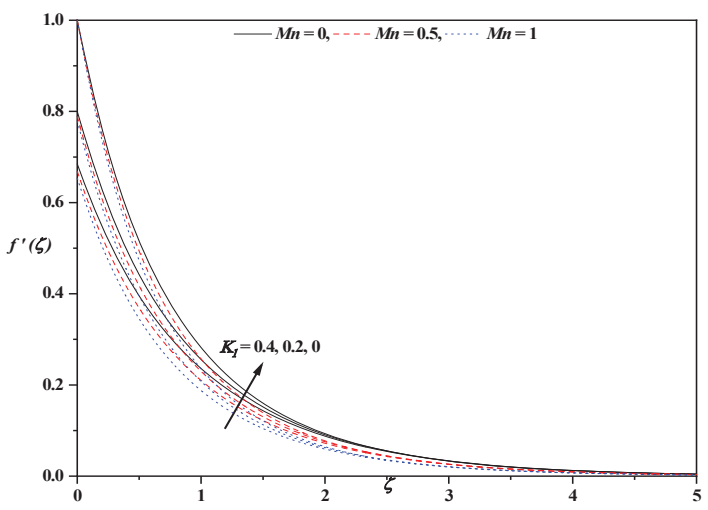

Fig. 5 Horizontal velocity profiles for different values of $M n$ and $K_{1}$ with $\operatorname{Pr}=1.09, S c=m=1, N b=B i=0.5, N t=\lambda=0.1$, $\mathrm{A}=\mathrm{B}=\beta=0.2$. 
Figure 6(a) demonstrates that the temperature profile $\theta(\zeta)$ which reduces for increasing values of Prand $\mathrm{A}$. Here, the increase in $\operatorname{Pr}(=v / \alpha)$ is responsible for lesser thermal diffusivity $\alpha$ which results in the reduced thermal boundary layer. A similar trend may be observed in the case of A. The impact of $N t$ and $B i$ on $\theta(\zeta)$ is sketched in Fig. 6(b). The terms $N t=\tau D_{T}\left(T_{w}-T_{\infty}\right) / v T_{\infty}$ and $B i=(h / k) \sqrt{2 v L / U_{0}}$ predicts the enhancement in temperature as both $N t$ and $B i$ increase. It is noticed that the fluid temperature is zero when $B i=0$ and it is prescribed temperature at the wall when it tends to infinity.

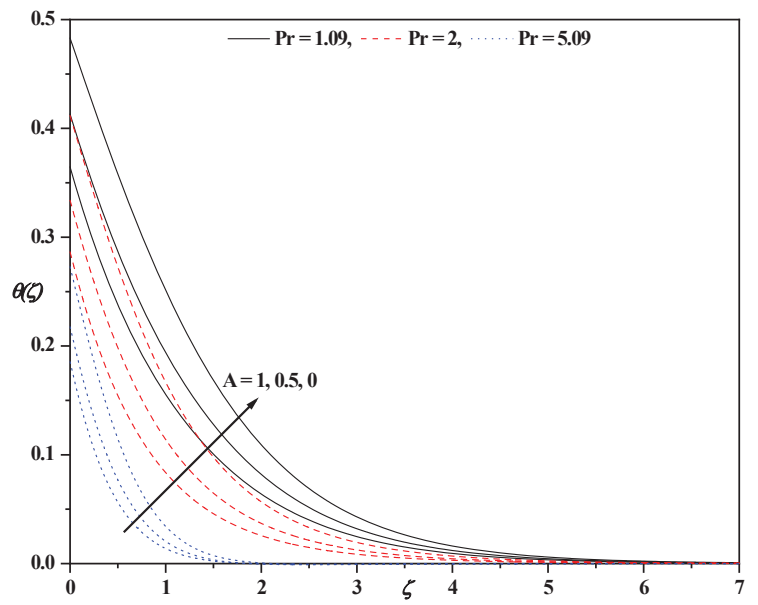

Fig. 6(a) Temperature profiles for different values of $\operatorname{Prand} \mathrm{A}$ with $S c=m=1, N b=B i=M n=0.5, N t=\lambda=0.1$, $\mathrm{B}=K_{1}=\beta=0.2$.

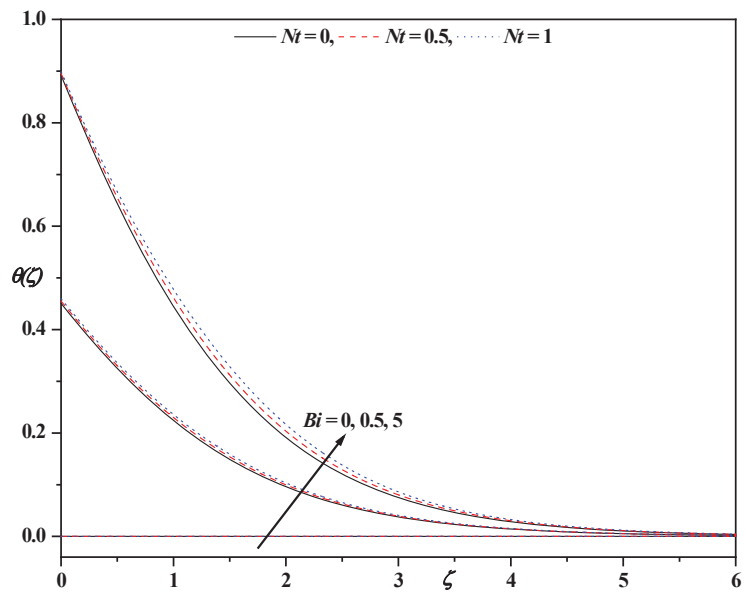

Fig. 6(b) Temperature profiles for different values of $N t$ and $B i$ with $\operatorname{Pr}=1.09, S c=m=1, N b=M n=0.5, \lambda=0.1$, $\mathrm{A}=\mathrm{B}=K_{1}=\beta=0.2$.

Figure 7(a) depicts the concentration profiles for different values of the Schmidt number $S c$ and concentration exponent parameter B. From the figure, it is clear that the rising values of both $S c$ and B enhances the concentration profile. The Schmidt number $S c$ shows the relative strength of the thermal diffusivity to the nanoparticle diffusion rate. The thermal diffusivity will exceed nanoparticle diffusivity when $S c>1$. This result stands for enhancement in the magnitudes of the nanoparticle concentration and thickened concentration boundary layer.

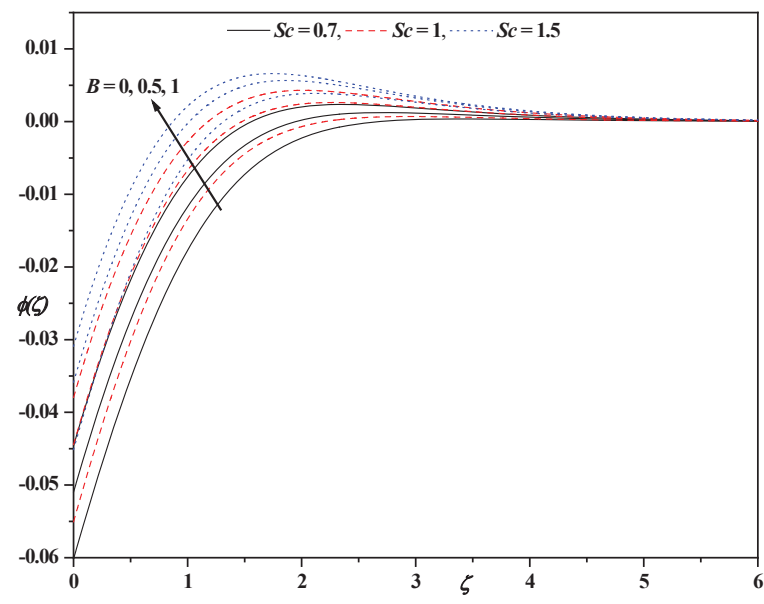

Fig. 7(a) Concentration profiles for different values of $S c$ and B with $\operatorname{Pr}=1.09, m=1, N b=B i=M n=0.5, N t=\lambda=0.1$, $\mathrm{A}=K_{1}=\beta=0.2$.

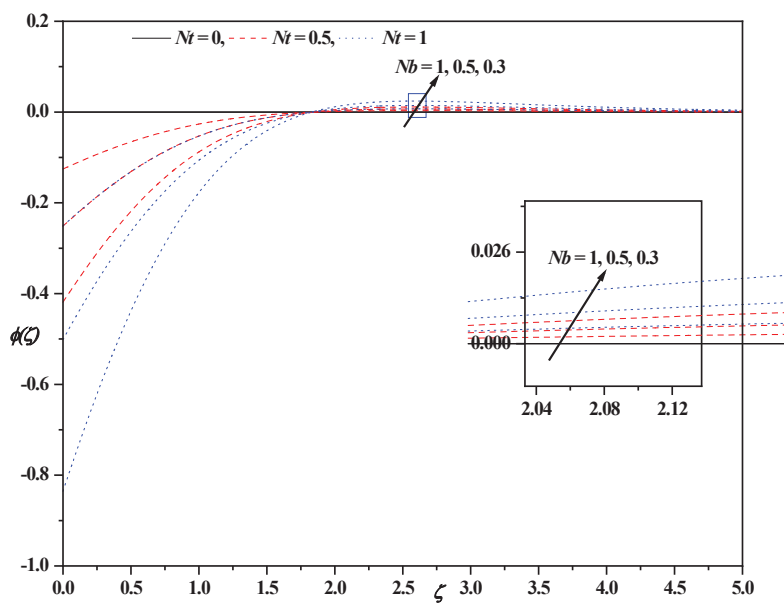

Fig. 7(b) Concentration profiles for different values of $N t$ and $N b$ with $\operatorname{Pr}=1.09, m=S c=1, B i=M n=0.5, \lambda=0.1$, $\mathrm{A}=\mathrm{B}=K_{1}=\beta=0.2$.

Figure 7(b) explains the impact of $N t$ and $N b$ on $\phi(\zeta)$. It is noted that the nanoparticle volume fraction increases with the increase in $N t$ (increase in thermophoresis force) and thus, an enhancement in the thickness of the concentration boundary layer is observed. In this case, the nanoparticles move away from the hot stretching sheet towards the cold ambient fluid under the influence of the temperature gradient. But in the case of $\mathrm{Nb}$ (smaller nanoparticles), the result is the opposite. However, $N b$ will stifle the diffusion of nanoparticles away from the surface, which results in a decrease in nanoparticle concentration values in the boundary layer. Finally, Figure $8(\mathrm{a}-\mathrm{c})$ displays the 3D plot of velocities and these plots exhibit similar results as that of velocity profiles.

Table 4 is tabulated to exhibit the influence of embedding parameters on the skin-friction coefficient, the local Nusselt number, and the local Sherwood number. It is seen that the rising values of $\lambda$ and $\beta$ decreases $f^{\prime \prime}(0), g^{\prime}(0)$ and $\phi^{\prime}(0)$ and increases $\theta^{\prime}(0)$. The effect of $m$ shows a quite opposite trend as compared with $\lambda$ and $\beta$. Further, $f^{\prime \prime}(0), \theta^{\prime}(0)$ and $\phi^{\prime}(0)$ are the decreasing function of $\mathrm{Mn}, \mathrm{A}, \mathrm{Pr}$ and B and increasing function of $K_{1}$ and $S c$. 
Table 4 The values of skin-friction, local Nusselt number and local Sherwood number for various physical parameters with $N t=0.1, N b=0.5$

\begin{tabular}{|c|c|c|c|c|c|c|c|c|c|c|c|c|c|c|c|c|c|c|c|c|c|c|}
\hline $\operatorname{Pr}$ & A & B & $S c$ & $B i$ & $\beta$ & $M n$ & $\lambda$ & $m$ & $K_{1}$ & $-f^{\prime \prime}(0)$ & $-h_{f}$ & $\hat{E}_{p}^{f}$ & $-g^{\prime}(0)$ & $-h_{g}$ & $\hat{E}_{p}^{g}$ & $-\theta^{\prime}(0)$ & $-h_{\theta}$ & $\hat{E}_{p}^{\theta}$ & $\phi^{\prime}(0)$ & $-h_{\phi}$ & $\hat{E}_{p}^{\phi}$ & $\begin{array}{l}\mathrm{CPU} \\
\text { time }\end{array}$ \\
\hline \multirow{3}{*}{1.09} & \multirow{3}{*}{0.2} & \multirow{3}{*}{0.2} & \multirow{3}{*}{1} & \multirow{3}{*}{0.5} & \multirow{3}{*}{0.2} & \multirow{3}{*}{0.5} & \multirow{3}{*}{0.1} & \multirow{3}{*}{1} & 0.0 & 1.50171 & 1.28150 & $2.12 \times 10^{-6}$ & 0.22358 & 1.39868 & $9.79 \times 10^{-6}$ & 0.28443 & 1.64350 & $3.12 \times 10^{-6}$ & $\begin{array}{c}0.0571 \\
3\end{array}$ & 1.36629 & $6.54 \times 10^{-7}$ & $624 \mathrm{~s}$ \\
\hline & & & & & & & & & 0.2 & 1.05565 & 1.15027 & $2.48 \times 10^{-6}$ & 0.18565 & 1.27430 & $1.29 \times 10^{-5}$ & 0.27388 & 1.54837 & $1.56 \times 10^{-6}$ & 0.05554 & 1.32737 & $1.09 \times 10^{-7}$ & $687 \mathrm{~s}$ \\
\hline & & & & & & & & & 0. & 0.85932 & 1.13670 & $2.08 \times 10^{-6}$ & 0.16337 & 1.26558 & $1.24 \times 10^{-5}$ & 0.26685 & 1.54884 & $2.86 \times 10^{-6}$ & 0.05383 & 1.30500 & $6.32 \times 10^{-8}$ & $651 \mathrm{~s}$ \\
\hline \multirow{3}{*}{1.09} & \multirow{3}{*}{0.2} & \multirow{3}{*}{0.2} & \multirow{3}{*}{1} & \multirow{3}{*}{0.5} & \multirow{3}{*}{0.2} & \multirow{3}{*}{0.5} & \multirow{3}{*}{0.1} & 1 & & 1.05565 & 1.15027 & $2.48 \times 10^{-6}$ & 0.18565 & 1.27430 & $1.29 \times 10^{-5}$ & 0.27388 & 1.54937 & $1.56 \times 10^{-6}$ & 0.05554 & 1.08737 & $1.09 \times 10^{-7}$ & $392 \mathrm{~s}$ \\
\hline & & & & & & & & 2 & 0.2 & 1.02295 & 1.18305 & $1.59 \times 10^{-6}$ & 0.18351 & 1.30220 & $6.47 \times 10^{-6}$ & 0.27519 & 1.58916 & $1.45 \times 10^{-6}$ & 0.05581 & 1.06671 & $1.78 \times 10^{-7}$ & $389 \mathrm{~s}$ \\
\hline & & & & & & & & 3 & & 1.01171 & 1.19386 & $1.35 \times 10^{-6}$ & 0.18289 & 1.31160 & $5.09 \times 10^{-6}$ & 0.27565 & 1.60237 & $1.47 \times 10^{-6}$ & 0.05589 & 1.06443 & $2.12 \times 10^{-7}$ & $398 \mathrm{~s}$ \\
\hline \multirow{3}{*}{1.09} & \multirow{3}{*}{0.2} & \multirow{3}{*}{0.2} & \multirow{3}{*}{1} & \multirow{3}{*}{0.5} & & & 0.0 & & & 1.04348 & 0.88977 & $2.90 \times 10^{-8}$ & 0.10493 & 1.27712 & $5.32 \times 10^{-6}$ & 0.27482 & 1.52442 & $1.13 \times 10^{-6}$ & 0.05569 & 1.05284 & $1.59 \times 10^{-7}$ & $319 \mathrm{~s}$ \\
\hline & & & & & 0.2 & 0.5 & 0.2 & 1 & 0.2 & 1.07905 & 1.22029 & $1.90 \times 10^{-5}$ & 0.46081 & 1.28995 & $5.18 \times 10^{-5}$ & 0.27208 & 1.59099 & $2.65 \times 10^{-6}$ & 0.05510 & 1.20626 & $7.04 \times 10^{-8}$ & $406 \mathrm{~s}$ \\
\hline & & & & & & & 0.4 & & & 1.21201 & 1.27865 & $1.13 \times 10^{-4}$ & 0.77021 & 1.04182 & $1.87 \times 10^{-5}$ & 0.26357 & 1.61183 & $8.99 \times 10^{-6}$ & 0.05318 & 1.45384 & $4.78 \times 10^{-8}$ & $373 s$ \\
\hline & & & & & & 0.0 & & & & 1.00033 & 1.20441 & $1.13 \times 10^{-6}$ & 0.18226 & 1.32105 & $3.97 \times 10^{-6}$ & 0.27613 & 1.61507 & $1.54 \times 10^{-6}$ & 0.05597 & 1.06449 & $2.51 \times 10^{-7}$ & $367 \mathrm{~s}$ \\
\hline 1.09 & 0.2 & 0.2 & 1 & 0.5 & 0.2 & 0.5 & 0.1 & 1 & 0.2 & 1.05204 & 1.13393 & $1.17 \times 10^{-6}$ & 0.29030 & 1.27572 & $3.55 \times 10^{-5}$ & 0.27415 & 1.54151 & $1.43 \times 10^{-6}$ & 0.05558 & 1.07645 & $1.21 \times 10^{-7}$ & $389 \mathrm{~s}$ \\
\hline & & & & & & 1.0 & & & & 1.09372 & 0.85781 & $2.22 \times 10^{-7}$ & 0.41054 & 1.22642 & $2.32 \times 10^{-4}$ & 0.27297 & 1.46064 & $1.50 \times 10^{-6}$ & 0.05525 & 1.09898 & $8.39 \times 10^{-8}$ & $388 \mathrm{~s}$ \\
\hline & & & & & 0.0 & & & & & 1.03520 & 1.34943 & $1.08 \times 10^{-5}$ & 0.43421 & 1.41332 & $2.22 \times 10^{-5}$ & 0.27349 & 1.69735 & $8.04 \times 10^{-6}$ & 0.05556 & 1.13296 & $9.48 \times 10^{-8}$ & $325 \mathrm{~s}$ \\
\hline 1.09 & 0.2 & 0.2 & 1 & 0.5 & 0.4 & 0.5 & 0.1 & 1 & 0.2 & 1.11821 & 1.10462 & $3.25 \times 10^{-5}$ & 0.48499 & 1.17409 & $1.08 \times 10^{-4}$ & 0.27146 & 1.45160 & $2.93 \times 10^{-6}$ & 0.05472 & 1.25205 & $7.17 \times 10^{-8}$ & $423 \mathrm{~s}$ \\
\hline & & & & & 0.8 & & & & & 1.18609 & 0.92313 & $8.35 \times 10^{-5}$ & 0.52801 & 0.98672 & $3.52 \times 10^{-4}$ & 0.27099 & 1.22764 & $4.78 \times 10^{-6}$ & 0.05442 & 1.15635 & $9.61 \times 10^{-8}$ & $361 \mathrm{~s}$ \\
\hline & & & & 0.0 & & & & & & 1.05204 & 1.13393 & $1.17 \times 10^{-6}$ & 0.29031 & 1.27572 & $3.55 \times 10^{-5}$ & 0.00000 & 0.00000 & 0.000000 & 0.00000 & 0.00000 & 0.00000 & $337 \mathrm{~s}$ \\
\hline 1.09 & 0.2 & 0.2 & 1 & 0. & 0.2 & 0.5 & 0.1 & 1 & 0.2 & 1.05204 & 1.13393 & $1.17 \times 10^{-6}$ & 0.2 & 1.27572 & $3.55 \times 10^{-5}$ & 0.27239 & 1.54605 & $1.63 \times 10^{-6}$ & 0.27647 & 1.07776 & $2.30 \times 10^{-6}$ & $378 \mathrm{~s}$ \\
\hline & & & & & & & & & & 1.05204 & 1.13393 & $1.17 \times 10^{-6}$ & 0.29031 & 1.27572 & $3.55 \times 10^{-5}$ & 0.52265 & 1.50517 & $7.95 \times 10^{-6}$ & 0.53300 & 1.18482 & $1.60 \times 10^{-5}$ & $379 \mathrm{~s}$ \\
\hline & & & & & & & & & & 1.05204 & 1.13393 & $1.17 \times 10^{-6}$ & 0.29031 & 1.27572 & $3.55 \times 10^{-5}$ & 0.27413 & 1.54089 & $1.44 \times 10^{-6}$ & 0.05527 & 1.24722 & $4.01 \times 10^{-8}$ & $408 \mathrm{~s}$ \\
\hline 1.09 & 0.2 & 0.2 & 1.0 & 0.5 & 0.2 & 0.5 & 0.1 & 1 & 0.2 & 1.05204 & 1.13393 & $1.17 \times 10^{-6}$ & 0.29031 & 1.27572 & $3.55 \times 10^{-5}$ & 0.27403 & 1.54088 & $1.40 \times 10^{-6}$ & 0.05543 & 1.14238 & $8.81 \times 10^{-8}$ & $369 \mathrm{~s}$ \\
\hline & & & 1.5 & & & & & & & 1.05204 & 1.13393 & $1.17 \times 10^{-6}$ & 0.29031 & 1.27572 & $3.55 \times 10^{-5}$ & 0.27390 & 1.54022 & $1.50 \times 10^{-6}$ & 0.05559 & 1.21553 & $1.58 \times 10^{-7}$ & $367 \mathrm{~s}$ \\
\hline & & 0.0 & & & & & & & & 1.05204 & 1.13393 & $1.17 \times 10^{-6}$ & 0.29031 & 1.27572 & $3.55 \times 10^{-5}$ & 0.27424 & 1.54163 & $1.41 \times 10^{-6}$ & 0.05562 & 1.06718 & $1.07 \times 10^{-7}$ & $357 \mathrm{~s}$ \\
\hline 1.09 & 0.2 & 0.5 & 1 & 0.5 & 0.2 & 0.5 & 0.1 & 1 & 0.2 & 1.05204 & 1.13393 & $1.17 \times 10^{-6}$ & 0.29031 & 1.27572 & $3.55 \times 10^{-5}$ & 0.27403 & 1.54088 & $1.46 \times 10^{-6}$ & 0.05543 & 1.14238 & $8.81 \times 10^{-8}$ & $369 \mathrm{~s}$ \\
\hline & & 1.0 & & & & & & & & 1.05204 & 1.13393 & $1.17 \times 10^{-6}$ & 0.29031 & 1.27572 & $3.55 \times 10^{-5}$ & 0.27390 & 1.53824 & $1.55 \times 10^{-6}$ & 0.05532 & 1.20167 & $2.51 \times 10^{-8}$ & $366 \mathrm{~s}$ \\
\hline & 0.0 & & & & & & & & & 1.05204 & 1.13393 & $1.17 \times 10^{-6}$ & 0.29031 & 1.27572 & $3.55 \times 10^{-5}$ & 0.25849 & 1.59760 & $2.77 \times 10^{-6}$ & 0.05229 & 1.27656 & $3.87 \times 10^{-8}$ & $352 \mathrm{~s}$ \\
\hline 1.09 & 0.5 & 0.2 & 1 & 0.5 & 0.2 & 0.5 & 0.1 & 1 & 0.2 & 1.05204 & 1.13393 & $1.17 \times 10^{-6}$ & 0.29031 & 1.27572 & $3.55 \times 10^{-5}$ & 0.29345 & 1.46311 & $2.23 \times 10^{-6}$ & 0.05885 & 1.31697 & $5.70 \times 10^{-7}$ & $361 \mathrm{~s}$ \\
\hline & 1.0 & & & & & & & & & 1.05204 & 1.13393 & $1.17 \times 10^{-6}$ & 0.29031 & 1.27572 & $3.55 \times 10^{-5}$ & 0.31724 & 1.35343 & $1.87 \times 10^{-6}$ & 0.06352 & 1.38964 & $9.52 \times 10^{-7}$ & $310 \mathrm{~s}$ \\
\hline 1.09 & & & & & & & & & & 1.05204 & 1.13393 & $1.17 \times 10^{-6}$ & 0.29031 & 1.27572 & $3.55 \times 10^{-5}$ & 0.29345 & 1.46311 & $2.23 \times 10^{-6}$ & 0.05885 & 1.31697 & $5.70 \times 10^{-7}$ & $361 \mathrm{~s}$ \\
\hline 2.00 & 0.2 & 0.2 & 1 & 0.5 & 0.2 & 0.5 & 0.1 & 1 & 0.2 & 1.05204 & 1.13393 & $1.17 \times 10^{-6}$ & 0.29031 & 1.27572 & $3.55 \times 10^{-5}$ & 0.33311 & 1.12825 & $1.10 \times 10^{-6}$ & 0.06652 & 1.44121 & $7.99 \times 10^{-6}$ & $368 \mathrm{~s}$ \\
\hline 5.09 & & & & & & & & & & 1.05204 & 1.13393 & $1.17 \times 10^{-6}$ & 0.29031 & 1.27572 & $3.55 \times 10^{-5}$ & 0.39126 & 0.97029 & $9.08 \times 10^{-9}$ & 0.07806 & 1.22071 & $4.25 \times 10^{-6}$ & $373 \mathrm{~s}$ \\
\hline
\end{tabular}




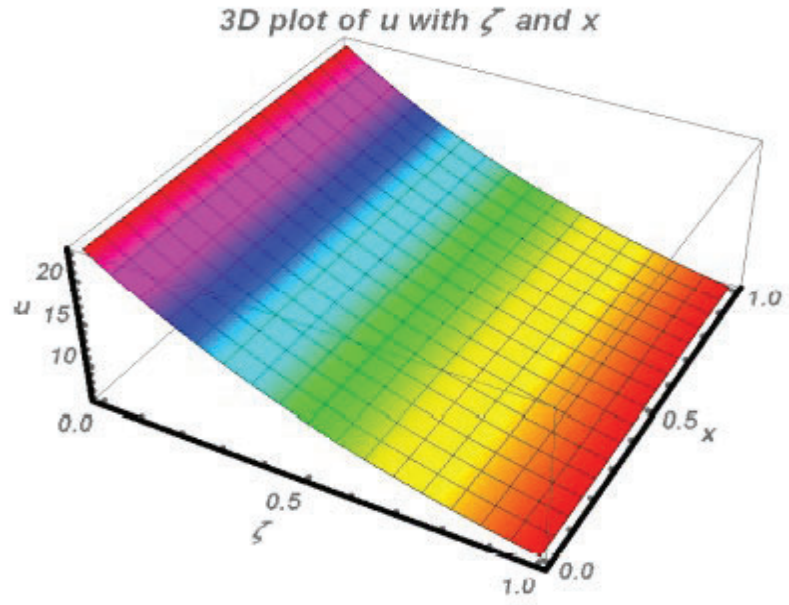

(a)

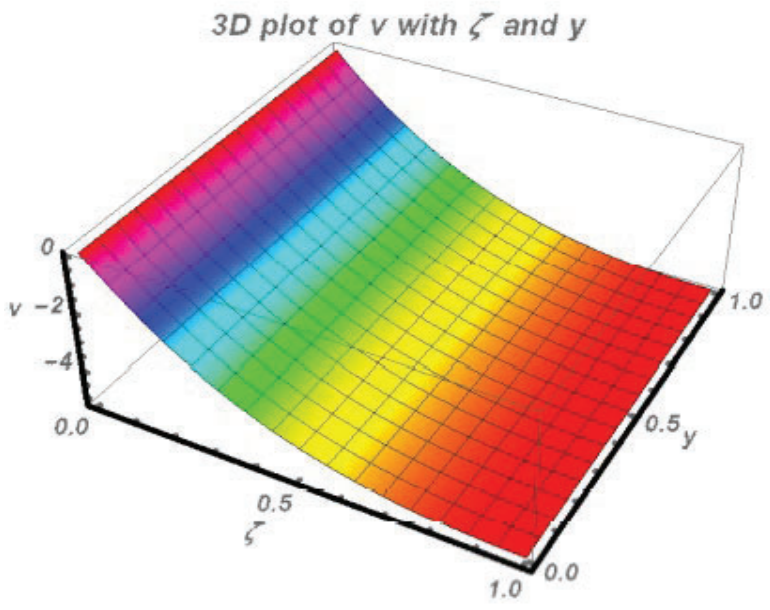

(b)

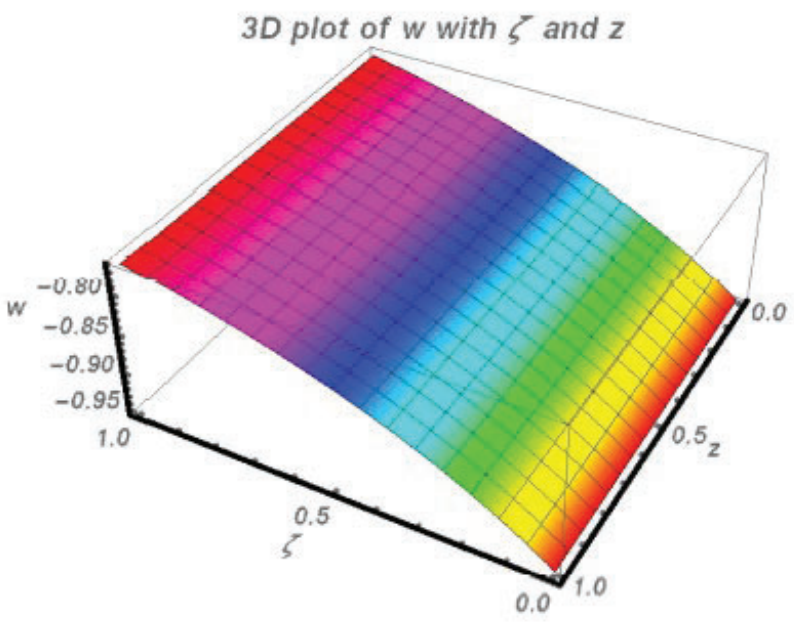

(c)

Fig. 8 Three dimensional plot of the velocity.

\section{CONCLUSIONS}

Some of the interesting findings of the present work are summarized below.

* The rotation parameter decreases the $f^{\prime}(\zeta), \mathrm{g}(\zeta), \theta(\zeta)$ and $\theta(\zeta)$ whereas the hall current parameter exhibits reverse trend.

* A substantial variation in Deborah number reduces $f^{\prime}(\zeta)$ and $\phi(\zeta)$ while $g(\zeta)$ and $\theta(\zeta)$ rises.
* The enhanced magnetic parameter and velocity slip parameter decreases $f^{\prime}(\zeta)$.

* Increased Prandtl number and temperature exponent and samller magnetic parameter, reduces $\theta(\zeta)$.

* An increase in $\phi(\zeta)$ is due to the increase in the Schmidt number, temperature exponent, thermophoresis parameter and the Brownian motion parameter.

\section{NOMENCLATURE}

temperature exponent parameter concentration exponent parameter magnetic induction vector Biots number magnetic field strength $\left[\mathrm{N} \mathrm{m}^{-1} \mathrm{~A}^{-1}\right]$ nanoparticles concentration skin friction co-efficient along $x$ and $y$ axis.

concentration at wall ambient fluid concentration Brownian diffusion coefficient $\left[\mathrm{m}^{2} \mathrm{~s}^{-1}\right]$ thermophoretic diffusion coefficient $\left[\mathrm{m}^{2} \mathrm{~s}^{-1}\right]$ electric charge intensity vector of the electric field dimensionless velocities heat transfer coefficient [ $\left.\mathrm{W} \mathrm{m}^{-2} \mathrm{~K}^{-1}\right]$ current density vector slip parameter thermal conductivity of fluid [ $\mathrm{W} \mathrm{m}^{-1} \mathrm{~K}^{-1}$ ] characteristic length

Hall effect parameter magnetic parameter electron number density electronic pressure Brownian motion parameter thermophoresis parameter local Nusselt number Prandtl number local Reynolds number Schmidt number local Sherwood number fluid temperature [K] hot fluid temperature ambient fluid temperature $[\mathrm{K}]$ reference velocity velocity components in the $x, y$ and $z$ directions $\left[\mathrm{m} \mathrm{s}^{-1}\right]$ stretching velocity in the $x$ and $y$ directions $\left[\mathrm{m} \mathrm{s}^{-1}\right]$.

\section{Greek symbols:}

$\begin{array}{ll}\alpha & \text { thermal diffusivity }\left[\mathrm{m}^{2} \mathrm{~s}^{-1}\right] \\ \beta & \text { Deborah number } \\ \lambda & \text { rotation parameter } \\ \lambda_{1} & \text { relaxation time }[\mathrm{s}] \\ \Omega & \text { constant angular velocity }\end{array}$


$\phi \quad$ dimensionless nanoparticles concentration

$v \quad$ kinematic viscosity $\left[\mathrm{m}^{2} \mathrm{~s}^{-1}\right]$

$\rho \quad$ fluid density $\left[\mathrm{kg} \mathrm{m}^{-3}\right]$

$\sigma \quad$ electrically conductivity $\left[\mathrm{S} \mathrm{m}^{-1}\right]$

$\theta \quad$ dimensionless temperature

$\tau \quad$ ratio between the effective heat capacity of the nanoparticle material and heat capacity of the fluid.

$\zeta \quad$ similarity variable

Subscripts:

$\infty \quad$ condition at infinity

$w \quad$ condition at wall

Superscript:

, differentiation with respect to $\zeta$.

\section{REFERENCES}

Amini, Y., Akhavan, S., and Izadpanah, E., 2020, "A numerical investigation on the heat transfer characteristics of nanofluid flow in a three-dimensional microchannel with harmonic rotating vortex generators." Journal of Thermal Analysis and Calorimetry, 139, 755764.

https://doi.org/10.1007/s10973-019-08402-6.

Animasaun, I. L., Mahanthesh, B., Jagun, A. O., Bankole, T. D., Sivaraj, R., Shah, N. A., and Saleem, S., 2019, "Significance of Lorentz Force and Thermoelectric on the Flow of $29 \mathrm{~nm} \mathrm{CuO-Water}$ Nanofluid on an Upper Horizontal Surface of a Paraboloid of Revolution." Journal of Heat Transfer, 141, 01-09.

https://doi.org/10.1115/1.4041971

Bidin, B., and Nazar, R., 2009, "Numerical solution of the boundary layer flow over an exponentially stretching sheet with thermal radiation." European Journal of Scientific Research, 33, 710-717.

Buongiorno, J., 2006, "Convective transport in nanofluids." $A S M E$, Journal of Heat Transfer, 128, 240-50.

https://doi.org/10.1115/1.2150834

Choi, S., 1995, "Enhancing Thermal Conductivity of Fluids with Nanoparticles." in Developments and Applications of Non-Newtonian Flows, D. A. Siginer, and H. P. Wang, eds., ASME, 231, 99-105.

Crane, L. J., 1970, "Flow past a stretching plate." Zeitschrift für angewandte Mathematik und Physik ZAMP, 21, 645-647. https://doi.org/10.1007/BF01587695

Elbashbeshy, E. M. A., 2001, "Heat transfer over an exponentially stretching continuous surface with suction". Archives of Mechanics.53, 643-651.

Fazle, M., Khan, W. A., and Ismail AI Md., 2017, "MHD flow over exponential radiating stretching sheet using homotopy analysis method." Journal of King Saudi University - Engineering Science. 29, 68-74.

$\underline{\text { http://dx.doi.org/10.1016/j.jksues.2014.06.001 }}$

Ganji, D. D., Jafarmadar, S., Jalilpour, B., Rahimi, R., Shotoban, A. B., and Hashemi, S. H. K., 2014, "Analytical and Numerical simulation investigation in effect of radiation and porosity on a nonorthogonal stagnation-point flow towards a stretching sheet." Indian Journal of Pure Applied Mathematics, 45, 415-432. https://doi.org/10.1007/s13226-014-0071-x

Grubka, L. J., and Bobba, K. M., 1985, "Heat transfer characteristics of a continuous, stretching surface with variable temperature." Journal of Heat Transfer, 107, 248-250.

https://doi.org/10.1115/1.3247387
Hayat, T., Muhammad, T., Shehzad, S. A., and Alsaedi, A., 2017, "Three dimensional rotating flow of Maxwell nanofluid." Journal of Molecular Liquids, 229, 495-500.

https://doi.org/10.1016/j.molliq.2016.12.095

Hayat, T., Awais, M., and Obaidat, S., 2012, "Three-dimensional flow of a Jeffery fluid over a linearly stretching sheet." Communications in Nonlinear Science and Numerical Simulation, 17, 699-707.

https://doi.org/10.1016/j.cnsns.2011.05.042

Lawrence, P. S., and Rao, B. N., 1992, "Heat transfer in the flow of a viscoelastic fluid over a stretching sheet." Acta Mechanica, 93, 5361.

https://doi.org/10.1007/BF01182572

Liao, S., 2010, “An optimal homotopy-analysis approach for strongly nonlinear differential equations." Communications in Nonlinear Science and Numerical simulation. 15, 2003-2016. https://doi.org/10.1016/j.cnsns.2009.09.002

Magyari, E., and Keller, B., 1999, "Heat and mass transfer in the boundary layers on an exponentially stretching continuous surface." Journal of Physical D: Applied Physics, 32, 577-585.

https://doi.org/10.1088/0022-3727/32/5/012

Majdi, H. Sh., Abdulkadhim, A., and Abed, A. M., 2019, "Numerical investigation of natural convection heat transfer in a parallelogramic enclosure having an inner circular cylinder using liquid nanofluid" Frointers Heat Mass Transf., 12, 2.

https://doi.org/10.5098/hmt.12.2.

Marinca, V., and Herisanu, N., 2015, "The Optimal Homotopy Asymptotic Method, Engineering Appllications, Springer. https://doi.org/10.1007/978-3-319-15374-2.

Mustafa, M., Wasim, M., Hayat, T., and Alsaedi, A., 2016, “A revised model to study the rotating flow of nanofluid over an exponentially deforming sheet: Numerical solutions." Journal of Molecular Liquids. 225, 320-327.

https://doi.org/10.1016/j.molliq.2016.11.078

Nadeem, S., Rizwan Ul Haq., Akbar, N. S., and Khan, Z. H., 2013, "MHD three-dimensional Casson fluid flow past a porous linearly stretching sheet." Alexandria Engineering Journal, 52, 577-582. https://doi.org/10.1016/j.aej.2013.08.005

Parand, K., Lotfi, Y., and Rad, J. A., 2017, "An accurate numerical analysis of the laminar two-dimensional flow of an incompressible Eyring-Powell fluid over a linear stretching sheet." European Physical Journal Plus, 132, 397-418. https://doi.org/10.1140/epjp/i2017-11693-3

Prasad, K. V., Vaidya, H., Vajravelu, K., and Ramanjini, V., 2018, "Analytical study of Cattaneo-Christov heat flux model for Williamson-nanofluid flow over a slender elastic sheet with variable thickness." Journal of nanofluid. 7, 583-594.

https://doi.org/10.1166/jon.2018.1475

Purusothaman, K., Murugesan, and Ali J Chamkha., 2019, "3D modeling of natural convective heat transfer from a varying rectangular heat generating source." Journal of Thermal Analysis and Calorimetry. 138, 597-608. https://doi.org/10.1007/s10973-019-08259-9.

Rahimi, J., Ganji, D. D., Khaki, and Hosseinzadeh, K., 2017, "Solution of the boundary layer flow of an Eyring-Powell nonNewtonian fluid over a linear stretching sheet by collocation method." Alexandria Engineering Journal. 56, 621-27.

https://doi.org/10.1016/j.aej.2016.11.006 
Rajagopal, K. R., Na, T. Y., and Gupta, A. S., 1984, "Flow of a viscoelastic fluid over a stretching sheet." Rheolgical Acta, 23, 213215.

https://doi.org/10.1007/BF01332078

Sajid, M., and Hayat, T., 2008, "Influence of thermal radiation on the boundary layer flow due to an exponentially stretching sheet." International Communication in Heat and Mass Transfer, 35, 347356.

https://doi.org/10.1016/j.icheatmasstransfer.2007.08.006

Sakiadis, B. C., 1961, "Boundary-layer behavior on continuous solid surfaces: I. Boundary-layer equations for two-dimensional and axisymmetric flow." American Institute of Chemical Engineers Journal, 7, 26-28.

https://doi.org/10.1002/aic.690070108

Srinivasacharya, D., and Jagadeeshwar, P., 2017, "Flow over an exponentially stretching sheet with hall, thermal radiation and chemical reaction effects," Frointers Heat Mass Transf., 9, 37. https://doi.org/10.5098/hmt.9.37

Swati Mukhopadhyay., 2013, "Slip effects on MHD boundary layer flow over an exponentially stretching sheet with suction/blowing and thermal radiation." Ain Shams Engineering Journal. 4, 485-491. https://doi.org/10.1016/j.asej.2012.10.007

Tiwari, R. K., and Das, M. K., 2007, "Heat transfer augmentation in two -sided lid driven differentially heated square cavity utilizing nanofluids." International Journal of Heat Mass Transfer. 50, 20022018.

https://doi.org/10.1016/j.ijheatmasstransfer.2006.09.034

Vaidya, H., Prasad, K. V., Vajravelu, K., Shehzad, S. A., and Hussain Basha., 2019a, "Role of Variable liquid properties in 3D flow of Maxwell nanofluid over convectively heated surface: Optimal solutions." Journal of nanofluid, 8, 1133-1146. https://doi.org/10.1166/jon.2019.1658

Vaidya, H., Prasad, K. V., Vajravelu, K., Makinde, O. D., and Srikantha setty, B., 2019b, "MHD Mixed convective flow of a casson nanofluid over a slender rotating disk with source/sink and partial slip effects." Defect and Diffusion Forum: Transfer phenomenon Fluid Heat Flows, 392, 158-177.

https://doi.org/10.4028/www.scientific.net/DDF.392.92

Van Gorder, R. A., 2019, "Optimal homotopy analysis and control of error for implicitly defined fully nonlinear differential equations." Numerical Algorithm. 81, 181-196.

https://doi.org/10.1007/s11075-018-0540-0

Weidman, P. D., and Ishak. A., 2015, "Multiple solutions of twodimensional and three-dimensional flows induced by a stretching flat surface." Communications in Nonlinear Science and Numerical Simulatio, 25, 01-09. https://doi.org/10.1016/j.cnsns.2015.01.003 